\title{
Ultrastructural alterations in the retinal pigment epithelium and photoreceptors of a Stargardt patient and three Stargardt mouse models: indication for the central role of RPE melanin in oxidative stress
}

\author{
Tatjana Taubitz ${ }^{\text {Corresp., }}{ }^{1}$, Alexander V Tschulakow ${ }^{1}$, Marina Tikhonovich ${ }^{1}$ ， Barbara Illing ${ }^{1}$, Yuan Fang ${ }^{1}$, \\ Antje Biesemeier $^{1}$, Sylvie Julien-Schraermeyer ${ }^{1}$, Ulrich Schraermeyer $^{1}$ \\ ${ }^{1}$ Division of Experimental Vitreoretinal Surgery, Centre for Ophthalmology, University of Tuebingen, Tuebingen, Germany \\ Corresponding Author: Tatjana Taubitz \\ Email address: tatjana.taubitz@med.uni-tuebingen.de
}

Background. Stargardt disease (SD) is characterized by the accumulation of the age-pigment lipofuscin in the retinal pigment epithelium (RPE) and subsequent neuroretinal degeneration. The disease leads to vision loss early in life. Here, we investigate age-dependent ultrastructural changes in three SD mouse models: albino $\mathrm{Abca} 4^{-/}$and pigmented $\mathrm{Abca} 4^{--}$and $\mathrm{Abca} 4^{-/} . \mathrm{Rdh}^{-/}$mice. Since we found indications for oxidative stress primarily in albino SD mice, we tested RPE melanin for its antioxidative capabilities.

Methods. SD mouse eyes were investigated by light, fluorescence and electron microscopy and were compared to the respective albino and pigmented wild type mice and to a human donor SD eye. To confirm the role of RPE melanin in scavenging oxidative stress, melanin from S. officinalis as a standard and porcine RPE were tested for their capability to quench superoxide anions.

Results. Histological alterations indicative of oxidative stress and/or lysosomal dysfunction were present in albino $\mathrm{Abca}^{-\%}$ and $\mathrm{Abca} 4^{-\%}$. Rdh8 ${ }^{-\%}$ mice. Retinal damage, such as inner segment rupture and pyknotic or free photoreceptor nuclei in the subretinal space and RPE vacuolization were exclusively found in albino Abca $4 \%$ mice. Shortened and disorganized photoreceptor outer segments and dead RPE cells were

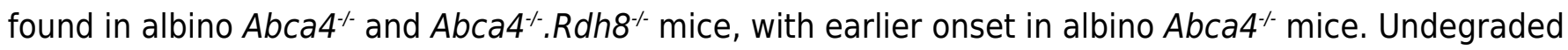
phagosomes and lipofuscin accumulation were present in the RPE of all three SD strains, but numbers were highest in $\mathrm{Abca}^{-\%}{ }^{-}$Rdh8 ${ }^{-}$mice. Lipofuscin morphology differed between SD strains: (melano)lipofuscin granules in pigmented $A b c a 4^{-/}$mice had a homogenous electron density and sharp demarcations, while lipofuscin in albino $A b c a 4^{-/}$mice had a flocculent electron density and often lacked a surrounding membrane, indicating loss of lysosomal integrity. Young $A b c a 4^{\%} \cdot R d h 8^{\%}$ mice showed (melano-)lipofuscin granules with homogenous electron density, while in aged animals granules with flocculent electron density predominated. Both strains of pigmented SD mice had melanolipofuscin clusters as found in the human SD eye. Like melanin from S. officinalis, also porcine RPE melanin can quench superoxide anions.

Discussion. The presented pathologies in albino $\mathrm{Abca} 4^{-/}$and $\mathrm{Abca} 4^{-/}$. Rdh $8^{-/}$mice suggest oxidative stress and/or lysosomal dysfunction within the RPE. Since albino Abca $4^{-/}$mice have the earliest onset and severest damage and as absence of melanin and also melanin turnover with age are known to diminish RPEs anti-oxidative properties, we assume that RPE melanin plays a role in SD related damages. A lack of pathology in pigmented $A b c a 4^{\%}$ mice due to lower stress levels as compared to the $A b c a 4^{\%}$. Rdh $8^{-\%}$ mice underlines this hypothesis. It is also supported by the finding that RPE melanin can quench superoxide anions. We therefore suppose that RPE melanin is important in retinal health and we discuss its role as 
an oxidative stress scavenger. 
1 Ultrastructural alterations in the retinal pigment epithelium and photoreceptors of a Stargardt

2 patient and three Stargardt mouse models: indication for the central role of RPE melanin in

3 oxidative stress

4

5 Tatjana Taubitz ${ }^{1}$, Alexander V. Tschulakow ${ }^{1}$, Marina Tikhonovich ${ }^{1}$, Barbara Illing ${ }^{1}$, Yuan

6 Fang ${ }^{1}$, Antje Biesemeier ${ }^{1}$, Sylvie Julien-Schraermeyer ${ }^{1}$, Ulrich Schraermeyer ${ }^{1}$

7

$8 \quad{ }^{1}$ Division of Experimental Vitreoretinal Surgery, Centre for Ophthalmology, University of

9 Tuebingen, Tuebingen, Germany

10

11

12

13

14 Corresponding author:

15 Tatjana Taubitz

16 Division of Experimental Vitreoretinal Surgery

17 Centre for Ophthalmology

18 University of Tuebingen

19 Schleichstrasse 12/1

2072076 Tuebingen, Germany

21 Phone: +4970712984888

22 Fax: +49 7071294554

23 Email: tatjana.taubitz@med.uni-tuebingen.de

24 


\section{Abstract}

26 Background. Stargardt disease (SD) is characterized by the accumulation of the age-pigment

27 lipofuscin in the retinal pigment epithelium (RPE) and subsequent neuroretinal degeneration.

28 The disease leads to vision loss early in life. Here, we investigate age-dependent ultrastructural 29 changes in three SD mouse models: albino $\mathrm{Abca4}^{-/-}$and pigmented $\mathrm{Abca4}^{-/-}$and $\mathrm{Abca}^{-/} . \mathrm{Rdh}^{-/-}$

30 mice. Since we found indications for oxidative stress primarily in albino SD mice, we tested RPE 31 melanin for its antioxidative capabilities.

32 Methods. SD mouse eyes were investigated by light, fluorescence and electron microscopy and 33 were compared to the respective albino and pigmented wild type mice and to a human donor SD 34 eye. To confirm the role of RPE melanin in scavenging oxidative stress, melanin from $S$.

35 officinalis as a standard and porcine RPE were tested for their capability to quench superoxide 36 anions.

37 Results. Histological alterations indicative of oxidative stress and/or lysosomal dysfunction were 38 present in albino $\mathrm{Abca} 4^{-/-}$and $\mathrm{Abca}^{-/-} \cdot \mathrm{Rdh}^{-/-}$mice. Retinal damage, such as inner segment 39 rupture and pyknotic or free photoreceptor nuclei in the subretinal space and RPE vacuolization 40 were exclusively found in albino $\mathrm{Abca}^{-/}$mice. Shortened and disorganized photoreceptor outer 41 segments and dead RPE cells were found in albino $\mathrm{Abca4^{-/ }}$ and $\mathrm{Abca4^{-/ }}$. $\mathrm{Rdh}^{-/-}$mice, with 42 earlier onset in albino $\mathrm{Abca4^{-/ }}$ mice. Undegraded phagosomes and lipofuscin accumulation were 43 present in the RPE of all three SD strains, but numbers were highest in $\mathrm{Abca4}^{-/} . \mathrm{Rdh}^{-/-}$mice.

44 Lipofuscin morphology differed between SD strains: (melano-)lipofuscin granules in pigmented $45 \mathrm{Abca}^{-/-}$mice had a homogenous electron density and sharp demarcations, while lipofuscin in 46 albino $\mathrm{Abca}^{-/-}$mice had a flocculent electron density and often lacked a surrounding membrane, 47 indicating loss of lysosomal integrity. Young $A b c a 4^{-/-} \cdot R d h 8^{-/-}$mice showed (melano-)lipofuscin 
48 granules with homogenous electron density, while in aged animals granules with flocculent

49 electron density predominated. Both strains of pigmented SD mice had melanolipofuscin clusters

50 as found in the human SD eye. Like melanin from S. officinalis, also porcine RPE melanin can

51 quench superoxide anions.

52 Discussion. The presented pathologies in albino $\mathrm{Abca4^{-/ }}$ and $\mathrm{Abca}^{-/-} \cdot \mathrm{Rdh}^{-/-}$mice suggest

53 oxidative stress and/or lysosomal dysfunction within the RPE. Since albino $A b c a 4^{-/-}$mice have

54 the earliest onset and severest damage and as absence of melanin and also melanin turnover with

55 age are known to diminish RPEs anti-oxidative properties, we assume that RPE melanin plays a

56 role in SD related damages. A lack of pathology in pigmented $\mathrm{Abca} 4^{-/-}$mice due to lower stress

57 levels as compared to the $A b c a 4^{-/} \cdot R d h 8^{-/-}$mice underlines this hypothesis. It is also supported by

58 the finding that RPE melanin can quench superoxide anions. We therefore suppose that RPE

59 melanin is important in retinal health and we discuss its role as an oxidative stress scavenger. 


\section{Introduction}

62 Stargardt disease (STGD1, OMIM \#248200) is one of the most frequent inherited macular

63 dystrophies in humans and affects 1 in 8,000-10,000 individuals (Blacharski 1988). It is caused

64 by mutations in the ATP-binding cassette A4 $(A B C A 4)$ gene, which encodes for a

65 transmembrane protein located in the rim of photoreceptor disks (Allikmets et al. 1997; Molday

66 et al. 2009). The ABCA4 protein is involved in all-trans retinal transport through the

67 photoreceptor disk membrane and is thus part of the visual cycle. Disrupted ABCA4 function

68 leads to accumulation of all-trans retinal within the disk and facilitates formation of N-

69 retinylidene-N-retinylethanolamine (A2E) and other bisretinoids (Sparrow et al. 2012). These

70 substances are major components of lipofuscin, a cytotoxic age pigment that accumulates in the

71 retinal pigment epithelium (RPE) and leads to RPE dysfunction and subsequent RPE and

72 photoreceptor degeneration (Sparrow et al. 2012). Owing to the burden of cytotoxic lipofuscin

73 accumulation early in life, Stargardt patients suffer from early onset macular degeneration,

74 resulting in progressive bilateral vision loss.

75 The first animal model generated for Stargardt disease was the pigmented $\mathrm{Abca}^{-/-}$mouse (Weng

76 et al. 1999). This model shows several typical pathological changes related to Stargardt disease,

77 including accumulation of lipofuscin granules in the RPE, elevated levels of A2E and other

78 bisretinoid fluorophores and delayed dark adaption (Charbel Issa et al. 2013). Since this mouse

79 strain lacks retinal degeneration (Charbel Issa et al. 2013; Weng et al. 1999), it is considered as a

80 model for the early stage of Stargardt disease.

81 A second $\mathrm{Abca}^{-/-}$mouse strain, the albino $\mathrm{Abca4^{-/- }}$ mouse, was generated by crossbreeding

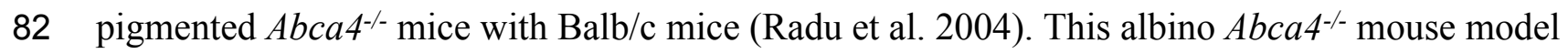

83 differs from the pigmented $\mathrm{Abca}^{-/-}$mouse model in several aspects. Albino $\mathrm{Abca4^{-- }}$ mice have 
84 lower levels of the lipofuscin component A2E, but higher levels of A2E oxidation products, so 85 called A2E oxiranes than pigmented $\mathrm{Abca}^{-/-}$mice (Radu et al. 2004). It was previously shown 86 that lipofuscin can generate reactive oxygen species, including singlet oxygen, after exposure to 87 blue light (Rozanowska et al. 1995) and that A2E is oxidized by singlet oxygen to A2E oxiranes 88 in vitro (Ben-Shabat et al. 2002). As a consequence, A2E can auto-oxidize to A2E oxiranes in 89 the presence of light. Since A2E oxiranes are highly reactive and can cause DNA fragmentation 90 (Sparrow et al. 2003a; Sparrow et al. 2003b), this might be an important mechanism of A2E 91 cytotoxicity. In contrast to pigmented $\mathrm{Abca4}^{-/-}$mice, albino $\mathrm{Abca4^{-/ }}$ mice show a mild retinal 92 degeneration, starting at an age of 7 months (Radu et al. 2011) and leading to a loss of approximately 30 to $40 \%$ of photoreceptor nuclei at the age of 11 months compared to WT

94 (Radu et al. 2008; Wu et al. 2010). Furthermore, albino $A b c a 4^{-/-}$mice show a range of signs of 95 chronic inflammation in the RPE including upregulated expression of oxidative stress genes, 96 elevated levels of the oxidative stress markers malondialdehyde and 4-hydroxynonenal, 97 activation of complement, downregulation of complement regulatory proteins and monocyte 98 chemoattractant protein-1, increased C-reactive protein immunoreactivity and thickening of 99 Bruch's Membrane (Radu et al. 2011).

100 A third Stargardt disease mouse model is the pigmented $\mathrm{Abca}^{-/-} \cdot \mathrm{Rdh}^{-/-}$mouse strain, generated 101 by crossbreeding $A b c a 4^{-/-}$mice with $R d h 8^{-/-}$mice (Maeda et al. 2008). $A b c a 4^{-/} \cdot R d h 8^{-/-}$mice are 102 described to have reduced all-trans retinal clearance and higher amounts of A2E, A2E-precursors 103 and retinal dimer conjugates than WT and pigmented $\mathrm{Abca4}^{-/-}$mice (Maeda et al. 2008).

104 Regional retinal degeneration and rosette formation is observed by as early as 6 weeks of age and 105 leads to advanced retinal degeneration by the age of 3 months (Maeda et al. 2008). Furthermore, 
106 drusen formation and thickening of Bruch's membrane in 3-month-old animals and choroidal

107 neovascularization in 10-month-old animals are described (Maeda et al. 2008).

108 So far, ultrastructural changes were not investigated in detail in all three mentioned Stargardt

109 disease mouse models, although they are widely used in the search of therapy options for SD. In

110 this study, we conducted light and fluorescence microscopic as well as ultrastructural analysis of

111 age-related retinal and RPE changes in these mouse strains. For comparison, we investigated

112 tissue of the respective WT mouse strains and a single eye of a human donor with Stargardt

113 disease. So far, electron microscopic examinations of human Stargardt eyes are scarce (Birnbach

114 et al. 1994; Bonilha et al. 2016; Eagle et al. 1980).

115 We found early onset of histologic alterations typical for oxidative stress and lysosomal

116 impairment in albino $A b c a 4^{-/}$mice. Similar alterations were found with later onset and less

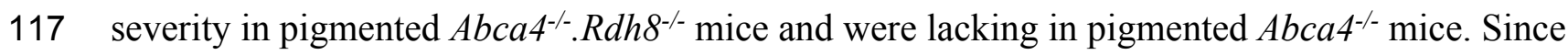

118 both the absence of melanin and melanin ageing are known to diminish the antioxidative

119 properties of the RPE, these finding underline the importance of RPE melanin for retinal health

120 and disease. 
121

122

123

124

125

126

127

128

129

130

131

132

133

134

135

136

137

138

139

140

141

142

143

\section{Materials and Methods}

2.1 Animals

Pigmented $\mathrm{Abca} 4^{-/-}$mice $\left(129 \mathrm{~S} 4 / \mathrm{SvJae}-\mathrm{Abca} 4^{\mathrm{tm} 1 \mathrm{Ght}}\right)$ were kindly donated by P. Charbel Issa (University of Oxford, Oxford, England). Albino $\mathrm{Abca}^{-/-}$mice (BALB/c-Abca4 ${ }^{\mathrm{tm} 1 \mathrm{Ght}}$ ) were kindly donated by G. Travis and R. Radu (University of California, Los Angeles, CA). Double knock-out $A b c a 4^{-/-} \cdot R d h 8^{-/-}\left(129 \mathrm{~S} 4 / \mathrm{SvJae}-\mathrm{Abca} 4^{\left.\mathrm{tm} 1 \mathrm{Ght} * \mathrm{C} 57 \mathrm{BL} / 6 \mathrm{~J}-\mathrm{Rdh} 8^{\mathrm{tm} 1 \mathrm{Kpal}}\right)}\right.$ mice were kindly donated by K. Palczewski (Case Western Reserve University, Cleveland, Ohio). Control albino WT mice (Balb/c) were purchased from Charles River (Sulzfeld, Germany) and control pigmented WT mice (129S2) were purchased from Harlan Laboratories (Hillcrest, UK). The knock-out mouse strains were bred in our in-house facility. Light cycling was $12 \mathrm{~h}$ light (approximately 50 lux in cages)/12h dark, food and water were available ad libitum. All procedures involving animals were in accordance with the German laws governing the use of experimental animals and were previously approved by the local agency for animal welfare (Einrichtung für Tierschutz, Tierärztlichen Dienst und Labortierkunde der Eberhard Karls Universität Tübingen, Tuebingen, Germany) and the local authorities (Regierungspräsidium Tübingen, Tuebingen, Germany).

\subsection{Sample preparation and light, fluorescence and electron microscopy}

Animals were sacrificed by carbon dioxide inhalation and subsequent cervical dislocation and the eyes were immediately enucleated.

For light and electron microscopy, eyes were fixed overnight at $4^{\circ} \mathrm{C}$ in $5 \%$ glutaraldehyde in 0.1 M cacodylate buffer ( $\mathrm{pH}$ 7.4). After washing in cacodylate buffer, the cornea and lens were removed and eye cups were hemisected. Halves were post-fixed in $1 \%$ osmium tetroxide in 0.1 
144 M cacodylate buffer and bloc-stained with uranyl acetate. Samples were dehydrated in a graded

145 series of ethanol and propylene oxide and embedded in Epon. Reagents were purchased from

146 AppliChem (Darmstadt, Germany), Merck (Darmstadt, Germany) and Serva (Heidelberg,

147 Germany). Light microscopy on toluidine blue stained semi-thin sections (500 nm) was

148 performed with a Zeiss Axioskop (Zeiss, Jena, Germany). Ultrathin sections (70 nm) were

149 mounted on copper slot grids (Plano, Wetzlar, Germany) and stained with lead citrate and

150 examined with a Zeiss 900 electron microscope (Zeiss, Jena, Germany).

151 For fluorescence microscopy and immunohistochemistry, eyes were fixed in $4.5 \%$ formaldehyde

152 (Carl Roth, Karlsruhe, Germany) and embedded in paraffin wax, cut into $5 \mu \mathrm{m}$ thick sections for

153 fluorescence microscopy and $4 \mu \mathrm{m}$ thick sections for immunohistochemistry. Samples for

154 fluorescence microscopy were deparaffinized according to standard procedures, cover-slipped

155 with FluorSave (Calbiochem, La Jolla, CA, USA) and were investigated with a Zeiss Axioplan2

156 imaging microscope (Zeiss, Jena, Germany). Lipofuscin autofluorescence was visualized with a

157 custom lipofuscin filter set (excitation $360 \mathrm{~nm}$, emission $540 \mathrm{~nm}$ ).

158

$159 \quad 2.3$ Immunohistochemistry

160 Sections were deparaffinized, rehydrated and subjected to heat induced antigen retrieval in a

161 pressure cooker for $2 \mathrm{~min}$ in either citrate buffer at $\mathrm{pH} 6$ (anti-HNE, anti-MDA) or Tris buffer at $162 \mathrm{pH} 9$ (anti-NITT). Sections were incubated overnight at $4^{\circ} \mathrm{C}$ with either rabbit anti-HNE (4-

163 hydroxy-2-noneal, HNE11-S, alpha diagnostic international; major product of endogenous lipid

164 peroxidation, 1:3500), rabbit anti-MDA (malondialdehyde, MDA11-S, alpha diagnostic

165 international; byproduct of endogenous lipid peroxidation, 1:2000) or rabbit anti-NITT 
166 (nitrotyrosine, NITT12-A, alpha diagnostic international; peroxynitrite-induced nitration of

167 tyrosine residues in proteins, 1:1000). Dilution of primary antibodies was done with

168 antibody diluent with background-reducing components (Dako S3022). The primary antibodies

169 were detected with the Dako REAL ${ }^{\mathrm{TM}}$ Detection System, Alkaline Phosphatase/RED,

170 Rabbit/Mouse according to the manufacturer's instructions. In brief, sections were incubated

171 with biotinylated goat anti-mouse and anti-rabbit immunoglobulins for $15 \mathrm{~min}$ at room

172 temperature with subsequent incubation with streptavidin conjugated to alkaline phosphatase for

$17315 \mathrm{~min}$ at room temperature. Reaction products were visualized with freshly prepared substrate

174 working solution supplemented with levamisole to block endogenous peroxidases. Sections were

175 counterstained with hematoxylin solution modified according to Gill III (Merck, Darmstadt,

176 Germany). To control for non-specific binding, control sections were prepared without

177 incubation with primary antibodies. Sections were investigated using a Zeiss Axioskop (Zeiss,

178 Jena, Germany).

179

180

2.4 Quantification of photoreceptor nuclei and outer segment length by light microscopy

181 Semi-thin sections of whole eye cups (up to $400 \mu \mathrm{m}$ from the optic nerve head) were

182 photographed using a $63 \mathrm{x}$ oil objective. Areas adjacent to the optic nerve and to the ora serrata

183 were excluded from analysis due to the physiological thinning of the retina.

184 Photoreceptor nuclei were counted semi-automatically in an average of 11 digital images per

185 section in on average 3 eyes per age group using Fiji software (Schindelin et al. 2012). In each

186 image, a region of interest with a width of $100 \mu \mathrm{m}$ along the outer nuclear layer was defined and

187 total photoreceptor nuclei within the region of interest were counted as number of photoreceptor

188 nuclei per $100 \mu \mathrm{m}$ width of retina. The total numbers of eyes investigated by light microscopy 
189 and used for photoreceptor nuclei quantification per strain were 34 eyes (albino $\mathrm{Abca}^{-/-}$mice),

19030 eyes (pigmented $\mathrm{Abca4^{-/- }}$ mice) and 47 eyes $\left(\mathrm{Abca}^{-/-} \cdot \mathrm{Rhh}^{-/-}\right.$mice).

191 Outer segment layer length was measured manually in $11-16$ positions per section in an average

192 of 2 eyes per age group using Fiji software.

193

2.5 Electron microscopic investigation

195 Whole sections of mouse eyes were thoroughly investigated by electron microscopy. Total

numbers of eyes investigated by electron microscopy for the different strains were 12 eyes

197 (pigmented $\mathrm{Abca4}^{-/-}$), 16 eyes (albino $\mathrm{Abca}^{4^{-/}}$), 16 eyes $\left(\mathrm{Abca}^{-/-} \cdot \mathrm{Rdh}^{-/-}\right.$), 6 eyes (albino WT)

198 and 3 eyes (pigmented WT). Sections were examined for changes in photoreceptor, RPE and

199 Bruch's membrane structure. Lipid droplets in RPE cells were counted and their diameter

200 measured in whole sections. Thickness of Bruch's membrane was measured in 15 consecutive

201 images. All measurements were performed with iTEM 5.0 Software (Olympus Soft Imaging

202 Solutions, Muenster).

203

204

2.6 Quantification of the area in RPE cytoplasm occupied by lipofuscin-like material

205

Lipofuscin-like material (derived from both lipofuscin and melanolipofuscin granules, hereafter 206 referred to as lipofuscin) was quantified in young (aged 4 to 6 months) and old (aged 12 months)

207 Stargardt mice in an average of 5 eyes per group. 30 electron micrographs of the RPE

208 (magnification $\mathrm{x} 20.000$ ) per eye were taken in a way so only cytoplasm and no nuclei, microvilli

209 and basal labyrinth were visible. To compensate for vignetting, only an oval region of interest in

210 the image center was analyzed. The Trainable Weka Segmentation (Arganda-Carreras et al.

211 2017) plugin for Fiji software, a machine learning tool, was used to determine the area of 
212 lipofuscin per image. A representative subset of images was used to train the tool to specifically

213 recognize lipofuscin, so melanin was excluded from the analysis. A combination of the mean,

214 median, maximum, minimum, variance and the anisotropic diffusion-, entropy- and neighbor

215 algorithms was used. Segmentation calculations were performed on a 16 virtual central

216 processing units system with 64 GB working memory (de.NBI Cloud Tübingen,

217 https://denbi.uni-tuebingen.de).

218

$219 \quad 2.7$ Human tissue

220 Glutaraldehyde-fixed perimacular tissue of a 72-year-old donor with clinically diagnosed

221 Stargardt disease was obtained through Foundation Fighting Blindness (Columbia, MD, USA).

222 Post mortem time until fixation was 48 hours. Written informed consent of the donor for use in

223 medical research and approval of the Institutional Review Board of the University of Tuebingen

224 (Ethik-Kommission an der Medizinischen Fakultät der Eberhard-Karls-Universität und am

225 Universitätsklinikum Tübingen, Tuebingen, Germany, approval number 462/2009BO2) were

226 obtained. The experiments were performed in adherence to the tenets of the Declaration of

227 Helsinki. Human tissue was embedded for standard electron microscopy as described above. For

228 investigation of lipofuscin autofluorescence in semi-thin sections, heavy-metal treatment during

229 embedding (osmium tetroxide, uranyl acetate) was omitted.

230

2312.8 Isolation of porcine RPE melanin

232 RPE melanin from pig eyes was isolated as described by (Boulton \& Marshall 1985) with

233 modifications (Zareba et al. 2006). In brief, pig eyes were opened close to the ora serrata and the

234 anterior segment and vitreous were discarded. The retina was removed with forceps and the eye 
235 cup washed with PBS (Gibco, Carlsbad, USA). Eye cups were filled with trypsin/EDTA (Gibco, 236 Carlsbad, USA) and incubated for $10 \mathrm{~min}$ at $37^{\circ} \mathrm{C}$. RPE cells were isolated by pipetting jet 237 streams onto the cell layer and collected in DMEM supplemented with 10\% fetal bovine serum 238 (Gibco, Carlsbad, USA) to stop the enzymatic reaction. RPE cell pellets were homogenized 239 using a tissue grinder. Cell debris were removed by centrifugation for $7 \mathrm{~min}$ at $60 \mathrm{~g}$ and the 240 obtained supernatant was centrifuged for $10 \mathrm{~min}$ at $6000 \mathrm{~g}$ to pellet all pigment granules. The 241 pellet was collected in $0.3 \mathrm{M}$ sucrose, loaded on a two-step sucrose gradient $(1 \mathrm{M}$ and $2 \mathrm{M})$ and 242 centrifuged for $1 \mathrm{~h}$ at $103.000 \mathrm{~g}$. Isolated RPE melanin granules were washed in PBS and the 243 final granule concentration was determined using a hemocytometer.

244

246 Quenching capability of melanin was investigated with the photochemical nitroblue tetrazolium 247 (NBT) assay (Cheng et al. 2015). In this assay, superoxide generated by illumination of 248 riboflavin reduces pale-yellow NBT to blue-violet formazan. The reaction mixture had a final 249 concentration of $0.1 \mathrm{mM}$ EDTA, $13 \mu \mathrm{M}$ methionine, $75 \mu \mathrm{M}$ NBT and $4 \mathrm{mM}$ riboflavin in PBS $250 \mathrm{pH}$ 7.4. EDTA, methionine, NBT and riboflavin were purchased from Sigma-Aldrich (St. Louis, 251 USA). The reaction mixture was illuminated for 15 minutes with blue light from an LED lamp at $2522.5 \mathrm{~mW} / \mathrm{cm}^{2}$ (450 nm, SunaEco 1500 Ocean Blue XP, Tropic Marin, Wartenberg, Germany). 253 Uniform illumination of the samples was ensured by placing the samples on a horizontally 254 aligned rotator. The formazan was quantified at $560 \mathrm{~nm}$ (Synergy HT, BioTek, Winooski, VT, 255 USA). 
256 Controls included positive controls (all reagents, no melanin, illuminated), negative controls (all 257 reagents, no melanin, kept in the dark) and blanks (no riboflavin, melanin, illuminated) to 258 compensate for melanin absorption at readout.

259

260 $2.10 \quad$ Statistical analysis

261 Statistical analysis was performed with JMP 13 (SAS, Cary, NC, USA). All data sets were tested 262 for normal distribution to decide on using parametric or non-parametric testing. Since all data 263 sets were not normally distributed and contained several groups, we used the Steel-Dwass All 264 Pairs test, a multiple non-parametrical test with alpha correction. The null hypothesis was that 265 the groups were not significantly different. Values are given as mean \pm standard deviation, $266 \mathrm{p}<0.05$ was considered statistically significant. 
267

268

269

270

271

272

273

274 275

276

277

278

279

280 281 pigmented WT.

282

283

284

285

286

287

288

289

290

\section{Results}

\subsection{Photoreceptors}

Light microscopic investigation of tissue of 12-month-old animals (Fig. 1 A) showed overall typical histology in pigmented $\mathrm{Abca4}^{-/}$mice compared to pigmented WT as already published in (Charbel Issa et al. 2013) (not shown). Albino $\mathrm{Abca}^{4-/}$ mice had shortened outer segments whereas $A b \mathrm{ca}^{-/-} \cdot R d h 8^{-/-}$showed a hypertrophy of the RPE (Fig. 1 A, Fig. S1).

Outer segment length was measured in light microscopy. Compared to age-matched albino WT animals, albino $\mathrm{Abca}^{-/-}$mice aged 4 months showed a significant reduction in outer segment length (albino $\mathrm{Abca}^{-/-} 4$ months $22.3 \pm 3.2 \mu \mathrm{m}$, albino WT 4 months $26.2 \pm 2.5 \mu \mathrm{m}, \mathrm{p}<0.001$, Fig. $1 \mathrm{~B})$. In $\mathrm{Abca4^{-/ }}$. $R d h 8^{-/-}$mice, outer segment shortening had later onset: No difference was found for 3-month-old animals, whereas 6-month-old animals had shortened outer segments $\left(\mathrm{Abca}^{-/-} . \mathrm{Rdh}^{-/-}\right.$3-month-old $32.1 \pm 2.7 \mu \mathrm{m}$, pigmented WT 3-month-old 32.6 $\pm 1.6 \mu \mathrm{m}$, not significant; $\mathrm{Abca4}^{-/-} . \mathrm{R}_{\mathrm{Ah}} \mathrm{8}^{-/-}$6-month-old $27.7 \pm 2.0 \mu \mathrm{m}$, pigmented WT 12-month-old $33.9 \pm 2.0$ $\mu \mathrm{m}, \mathrm{p}<0.0001$, Fig. 1 B). Pigmented $\mathrm{Abca4}^{-/}$had no outer segment shortening compared to

A detailed analysis of the age-dependent reduction of photoreceptor nuclei numbers of the three different Stargardt mouse strains revealed similar retinal degeneration courses for both albino

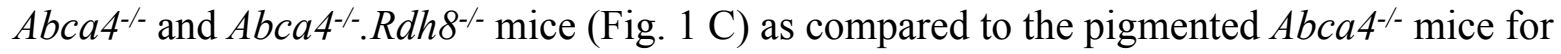
which it is known that they do not present signs of retinal degeneration (Charbel Issa et al. 2013; Weng et al. 1999). We quantified total photoreceptor nuclei numbers, as opposed to the more commonly used outer nuclear layer thickness, to account for nuclei disorganization and gaps between nuclei that occurred in albino $\mathrm{Abca4}^{-/-}$and, to a lesser extent, in $\mathrm{Abca4^{-/ }} \cdot \mathrm{Rdh}^{-/-}$mice.

On the ultrastructural level, shrunken, electron dense nuclei, resembling pyknosis, were only found in albino $\mathrm{Abca}^{-/}$animals (Fig. $\left.1 \mathrm{D}\right)$. Furthermore, isolated photoreceptor nuclei were 
291 present in the subretinal space next to unusually thinned RPE cells, even in young albino Abca4 $4^{-/}$ 292 animals (Fig. 1 E). In our colony of $A b c a 4^{-/} \cdot R d h 8^{-/}$mice, we could not find the previously 293 described rosette formation at 3 weeks and the rather prominent retinal degeneration beginning at 2943 months (Maeda et al. 2008). Rosette formation was only seen in a single 12-month-old animal. 295 Instead, RPE hypertrophy was regularly seen in $A b c a 4^{-/} \cdot R d h 8^{-/}$animals (Fig. 1 A, Fig. S1). 296 Genotype and age-dependent alterations of the disc membrane stacking were apparent on the 297 ultrastructural level (Fig. 2 A, Table 1). Those alterations seemed to start at the outer segment 298 tips (next to the RPE) and spread towards the base of the outer segment. Alterations in outer 299 segment tip organization were never seen in pigmented WT and pigmented $A b c a 4^{-/}$mice. In 300 albino WT and $A b c a 4^{-\digamma} \cdot R d h 8^{-/}$animals, it was not seen in the youngest animals investigated (3-4 301 months), but in animals aged 6-7 months and older. In albino $A b c a 4^{-/}$mice, outer segment tip 302 disorganization was already present in 4-month-old animals, the youngest group investigated in 303 this strain.

304 In albino $A b c a 4^{-/}$and $A b c a 4^{-/} \cdot R d h 8^{-/}$animals, alterations in overall disc membrane stacking 305 were observed starting with 6 months but were lacking in pigmented and albino WT and 306 pigmented $\mathrm{Abca4}^{-/}$animals.

307 Inner segments of photoreceptors appeared swollen and/or ruptured in albino Abca4 $4^{-/}$mice (Fig. $3082 \mathrm{~B})$. In pigmented $A b c a 4^{-/}$mice and $A b c a 4^{-/} . R d h 8^{-/}$mice, inner segment damages were only 309 occasionally observed (Table 1).

310 In albino $\mathrm{Abca}^{-/}$mice, agglomerated electron dense material was occasionally present between 311 inner segment and outer segment (Fig. 2 C) and disk membrane renewal seemed to be somewhat 312 disturbed as disk membrane swirls were sporadically present at the nascent part of the outer 
313 segment (Fig. 2 D). These changes were not present in albino WT and pigmented Stargardt mice.

314 Outer segment tips were in contact to RPE microvilli in all mouse strains and age groups.

315

$316 \quad 3.2$ Vacuole-like structures within the RPE

317 In semi-thin sections of albino $A b c a 4^{-/}$mice, vacuole-like structures within the RPE monolayer

318 were apparent in animals as young as 6 months (Fig. 3 A). Electron microscopic investigation

319 revealed that the vacuole-like structures were an enlargement of the intercellular space between

320 RPE cells and were apically limited by the junctional complex (Fig. 3 B). Sometimes vacuole-

321 like structures were also seen within the cytoplasm, usually in proximity to the lateral plasma

322 membrane and probably also of intercellular space origin. The contents of the vacuole-like

323 structures varied between empty (not shown), filled with membranous material (Fig. 3 B) and

324 filled with a variety of fibrillous, granular, lamellar and amorphous materials (Fig. 3 C).

325 Different types of contents were often seen within the same eye. Ultrastructurally, vacuole-like

326 structures were apparent in albino $A b c a 4^{-/}$mice of all age groups, but were barely present in

327 pigmented $\mathrm{Abca}^{-/}$and $A b c a 4^{-/} . R d h 8^{-/}$mice (Fig. $3 \mathrm{D}$ ). The number of vacuole-like structures

328 increased with age, though there was a considerable variance between eyes of the same age.

329 Vacuole-like structures were considerably fewer in albino WT mice.

\subsection{Lipofuscin}

332 To compare the lipofuscin autofluorescence between the different Stargardt mouse strains, 333 paraffin sections of 12-month-old animals were investigated by fluorescence microscopy (Fig. 4

334 A). In pigmented $\mathrm{Abca}^{-/}{ }^{-}$sections, the lipofuscin autofluorescence was clearly defined, 335 indicating the presence of granules with well-defined borders. In albino Abca4 $4^{-/}$sections, the 
336 lipofuscin autofluorescence was predominantly blurry. In $A b c a 4^{-/} . R d h 8^{-/}$sections, both clearly

337 defined and blurry lipofuscin autofluorescence were present. Autofluorescence in albino Abca4 $4^{-1}$

338 and $\mathrm{Abca}^{-1-} \cdot \mathrm{Rdh}^{-/-}$tissue was elevated compared to pigmented $\mathrm{Abca4^{-/ }}$ tissue.

339 The morphological appearance of lipofuscin granules in the different Stargardt mouse strains was 340 investigated by electron microscopy. Typical morphological appearances are illustrated in Fig. 4

341 B and age-dependent quantification of the different identified lipofuscin subtypes can be found 342 in Table 1.

343 In pigmented $\mathrm{Abca}^{-/}$mice, we found exclusively lipofuscin with homogenous electron density 344 and clearly defined margins indicating an intact limiting membrane (Fig. 4 B), as previously

345 described (Charbel Issa et al. 2013). Most of the lipofuscin was fused to melanin and formed 346 melanolipofuscin.

347 In albino $\mathrm{Abca}^{-/}$mice, lipofuscin with homogenous electron density was also seen. However,

348 the majority of lipofuscin granules belonged to another type that had a flocculent electron density

349 (Fig. 4 B, Table 1). The margins of this type of lipofuscin were often not clearly defined,

350 suggesting loss of the lysosomal membrane. As albino $\mathrm{Abca}^{-/}$mice accumulated lipofuscin with 351 age, the amount of lipofuscin with flocculent electron density increased while the amount of

352 lipofuscin with homogenous electron density seemed to stay constant (Table 1).

353 In $A b c a 4^{-/} . R d h 8^{-/}$mice, the first type of lipofuscin with homogenous electron density had a

354 considerable variability between age groups (Table 1). After the age of 12 months, the second 355 type of lipofuscin with flocculent electron density was also present (Table 1). As in pigmented 356 Abca4 $4^{-/}$mice, most of the lipofuscin was fused to melanin and formed melanolipofuscin.

357 Membranes enclosing lipofuscin of the flocculent electron density type were often damaged or

358 absent (Fig. 4 C). In very old animals (aged 23 months and older), the RPE cytoplasm was 
359 abnormally electron dense (Fig. 4 D). Clear demarcations between lipofuscin granules and the 360 cytoplasm were hardly visible.

361 The area of RPE cytoplasm occupied by lipofuscin (both derived from lipofuscin and

362 melanolipofuscin granules) was quantified in electron micrographs of young (4-6 months) and 363 old (12 months) Stargardt mice (Fig. 5). In both age groups, $A b c a 4^{-/}$. $R d h 8^{-/-}$mice had the highest

364 levels of lipofuscin (young $16.9 \% \pm 8.3 \%$, old $26.9 \% \pm 5.3 \%$ ). In young animals, albino $\mathrm{Abca}^{\mathrm{4}^{-/}}$

365 mice had almost twice the levels of pigmented $\mathrm{Abca}^{-/-}$mice (albino $\mathrm{Abca}^{-/} 7.5 \% \pm 3.9 \%$,

366 pigmented $\left.\mathrm{Abca4}^{-/} 3.9 \% \pm 2.7 \%, \mathrm{p}<0.0001\right)$. In 12-month-old animals, the difference of

367 lipofuscin levels in albino and pigmented $\mathrm{Abca}^{-/-}$mice was lower but nevertheless statistically

368 significant (albino $\mathrm{Abca}^{-/-} 11.0 \% \pm 5.5 \%$, pigmented $\mathrm{Abca4^{-/ }} 9.3 \% \pm 5.5 \%, \mathrm{p}<0.05$ ).

369

370

371

372

373

374

375

376

377

378

379

380

\subsection{Lipid droplets}

Lipid droplets were frequently seen in RPE cells of all investigated mouse strains, especially in albino $\mathrm{Abca}^{4^{-/}}$mice (Table 1). In pigmented and albino WT animals, lipid droplets were small (median diameter: albino WT $235 \mathrm{~nm}$, pigmented WT $353 \mathrm{~nm}$ ) and the majority of droplets was fused to lipofuscin (albino WT 59\% and pigmented WT 82\% of total lipid droplets, Fig. S2). They were found in both apical and basal parts of the RPE cells, with a tendency to be more often apically located (Table 1). In Stargardt mouse strains, lipid droplets were bigger (median diameter: pigmented $A b c a 4^{-/} 555 \mathrm{~nm}$, albino $A b c a 4^{-/-} 705 \mathrm{~nm}, A b c a 4^{-/} . R d h 8^{-/-} 687 \mathrm{~nm}$ ) and only sporadically seen in the apical part of the RPE (Table 1, Fig. S2). Fusion with lipofuscin was rarely seen in albino $\mathrm{Abca}^{-/-}$mice (3\% of total lipid droplets) and absent in pigmented Stargardt mice. 
3.5 RPE integrity and Bruch's membrane

383

384 385 386 387 388 389 390 391 392 393 394 395 396

397 398 399 400 401 402 403 404

Dead RPE cells were regularly encountered in eyes of albino $\mathrm{Abca}^{-/-}$mice of all ages (Fig. 6 A, Table 1). In contrast, dead RPE cells were only found in aged $\mathrm{Abca}^{-/-} \cdot \mathrm{R} \mathrm{dh} \mathrm{8}^{-/-}$mice (12 months and older). In pigmented $\mathrm{Abca}^{-/-}$and young $\mathrm{Abca}^{4^{-/}} \cdot \mathrm{Rdh}^{-/-}$mice, dead RPE cells were not found. Locally, RPE cells were unusually thin in albino $A b c a 4^{-/-}$mice (Fig. 1 E).

RPE cells regularly contained undegraded phagosomes in the basal area of the cytoplasm in all three Stargardt mouse strains (Table 1). RPE cells, whose cytoplasm was largely occupied by extensive undegraded phagosomes, were found in albino $\mathrm{Abca}^{-/-}$and $\mathrm{Abca}^{-/-} \cdot \mathrm{Rdh}^{-/-}$mice (Fig. $6 \mathrm{~B})$. Highest numbers of undegraded phagosomes were found in $\mathrm{Abca4^{-/ }}$. $R d h 8^{-/-}$mice (Table 1). Irregularly shaped basal infoldings were frequently seen in albino $\mathrm{Abca} 4^{-/-}$mice, but rarely in pigmented Stargardt mice (Table 1). The irregular shape was due to the basal infoldings expanding to the lateral side of the cells (Fig. 6 C). In general, basal infoldings were reduced in number and expansion in all Stargardt mouse strains compared to wild types (Fig. 6 D). Bruch's membrane became thicker with age in all Stargardt mouse strains (Fig. 7). There was no significant difference between the different strains.

3.6 Histopathology and pigment changes in late Stargardt disease

For comparison, we also investigated a single eye of a 72-year-old Stargardt patient (Fig. 8). In this late stage of the disease, photoreceptors were almost completely degenerated resulting in geographic atrophy as is typical for Stargardt patients (Fig. 8 A). A reliable quantification of neuroretinal changes was not possible due to the long death-to-fixation time of the donor, but pigments can still be investigated under these conditions. RPE cells were nearly completely filled with pigment granules (Fig. 8 B), mostly lipofuscin and melanolipofuscin, melanin granules 
405 were barely present. Microvilli and basal infoldings were absent, while Bruch's membrane was 406 unorganized and contained electron dense deposits (Fig. 8 B). Melanolipofuscin formed clusters 407 (Fig. $8 \mathrm{C}, \mathrm{D}$ ), as they were also found in pigmented $\mathrm{Abca4^{-/ }}$ and $\mathrm{Abca4^{-/ }}$. $\mathrm{Rdh}^{\mathrm{O}^{-/-}}$mice.

408

409

410 Since ultrastructural changes in the RPE of Stargardt mice were indicative of oxidative stress, we 411 tested for oxidative stress markers in 12-month-old mice by immunohistochemistry. We found 412 HNE, MDA and NITT adducts in the RPE cells of pigmented and albino $\mathrm{Abca4^{-/ }}$ and $\mathrm{Abca4^{-/- }}$ $413 . R d h 8^{-/-}$mice with signals being generally lowest in pigmented $\mathrm{Abca4^{-/ }}$ mice (Fig. 9). HNE, 414 MDA and NITT signals were also present in the neuroretina, reaching from the inner limiting 415 membrane to the inner segments, in all Stargardt strains and were highest in $\mathrm{Abca}^{-/-} . \mathrm{Rdh}^{-/-}$mice 416 in the case of HNE and MDA or at similar levels in the three strains in case of NITT (Fig. S3).

3.8 RPE melanin can quench superoxide

419 To investigate the capability of RPE melanin to quench superoxide, we employed the 420 colorimetric NBT assay (Cheng et al. 2015). We first tested melanin from S. officinalis as a 421 melanin standard for its quenching capabilities and found a dose-response relationship (Fig. 10

422 A). Subsequently, we investigated RPE melanin isolated from pig eyes and found an 423 approximately $30 \%$ reduction of detectable superoxide for $3 \times 10^{4}$ RPE melanin granules utilized 424 in the assay (Fig. 10 B). 
426 The goal of this study was a comparison of the age-dependent ultrastructural changes in three

427 frequently used mouse models for Stargardt disease in order to better understand these models.

428 The three strains differ in their genetic background: pigmented $\mathrm{Abca}^{-/}$mice are bred on a 129

429 background, albino $\mathrm{Abca4^{-/ }}$ mice have a BALB/c background and pigmented $\mathrm{Abca}^{4^{-/}} \cdot \mathrm{Rdh}^{-/-}$

430 mice have a mixed 129.B6 background.

431 Both the 129 and BALB/c strains have the Leu450 variant of RPE65, while the B6 strain has the

432 Met450 variant, resulting in a lower visual cycle turnover and reduced light damage

433 susceptibility in this strain. Since the pigmented $\mathrm{Abca} 4^{-/} \cdot \mathrm{Rdh}^{-/-}$strain is on a mixed 129.B6

434 background, both alleles of RPE65 are present in this strain. Retinal degeneration was reported to 435 be more common in $A b c a 4^{-/} \cdot R d h 8^{-/-}$mice carrying the Leu450 variant $(97.5 \%$ of investigated 436 eyes) than animals carrying the Met450 variant (43.8\% of investigated eyes) (Maeda et al. 2008).

437 We did not genotype our animals for RPE65.

438 There is a growing body of evidence that different WT mouse strains show varied susceptibility 439 to light-induced damage that is brought on by so far unidentified genetic modulators. For 440 instance, even though BALB/c and 129 mice both have the LEU450 variant of RPE65, resulting 441 in a high visual cycle turnover, BALB/c mice show a lower resistance to light-induced retinal

442 damage that is independent of pigmentation (Danciger et al. 2004). Instead, two significant and 443 two suggestive quantitative trait loci were identified that might be the reason for the greater 444 sensitivity to light damage in BALB/c mice (Danciger et al. 2004). These findings were gained 445 from experiments using intense, short-term light exposure, but their relevance for moderate, 446 long-term light exposure as employed in animal husbandry is currently unknown. Nevertheless, 
447 care must be taken when comparing the results from the three different Stargardt mouse models

448 concerning their predisposition to retinal degeneration.

449

450

451

452

453

454

455

456

457

458

459

460

461

462

463

464

465

466

467

468

469

4.1 Among Stargardt mice, albino $\mathrm{Abca}^{-/-}$mice show the earliest onset and most varied retinal damages

It was previously described that albino $\mathrm{Abca4}^{-/-}$mice show a mild retinal degeneration that is not present in albino WT mice (Sparrow et al. 2013; Wu et al. 2010). We found that absolute photoreceptor nuclei numbers decrease with age, resulting in a steeper decline of photoreceptor

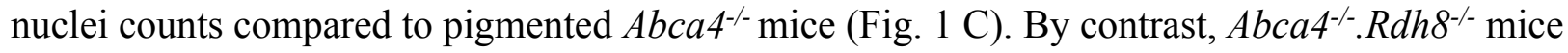
are described to have regional retinal degeneration and rosette formation as early as 6 weeks of age and advanced retinal degeneration by 3 months (Maeda et al. 2008). We were unable to reproduce the published results with our colony of $A b c a 4^{-/-} \cdot R d h 8^{-/-}$mice. Our animals had a similar rate of retinal degeneration than the albino $\mathrm{Abca}^{-/-}$mice (Fig. $1 \mathrm{C}$ ) and rosette formation was only found in one 12-month-old animal from a total of $25 \mathrm{Abca4^{-/ }}$. $R d h \mathrm{~S}^{-/-}$animals examined light-microscopically up to an age of 25 months.

In addition to the photoreceptor loss, albino $\mathrm{Abca}^{-/-}$mice show outer segment shortening (Fig. 1 B), disordered disk membranes (Fig. 2 A), damaged inner segments (Fig. 2 B), and morphological changes at the base of the outer segments (Fig. 2 C, D). These damages were not seen in pigmented $\mathrm{Abca}^{-/-}$and to a much lesser extent in $\mathrm{Abca4^{-/ }} \cdot \mathrm{Rdh}^{-/-}$mice (Table 1).

Shortening and disorganization of outer and inner segments was also described after conditional knockout of superoxide dismutase in the RPE, resulting in oxidative stress (Mao et al. 2014). In Stargardt patients, the junction between the inner and outer segments was described to be unorganized and/or lost on SD-OCT (Gomes et al. 2009). Furthermore, photoreceptor anomalies 
470 were found in patients that did not display equivalent fundus autofluorescence anomalies (Gomes

471 et al. 2009). Damaged inner segments and defective outer segment synthesis in albino $\mathrm{Abca4^{-/- }}$

472 mice (Fig. 2 B-D), but not in pigmented Stargardt mouse strains, might be histological

473 representations of the SD-OCT findings in Stargardt patients and needs further investigation.

474 Outer segment renewal starts at the base of the outer segment, so the disks at the tips are the

475 oldest disks in a photoreceptor and are affected the longest by any disturbance, e.g. accumulation

476 of retinoids due to lack of ABCA4. Loss of fundamental outer segment proteins like rhodopsin

477 (Lem et al. 1999) or GARP (Huttl et al. 2005) leads to a severe outer segment damage starting

478 early in life (reviewed by (Goldberg et al. 2016)). ABCA4 is located in the rims of disk

479 membranes (Papermaster et al. 1978) and does not form stable interactions with the peripherin-

480 2.GARP complex (Poetsch et al. 2001), which is essential for linking disk rims to the outer

481 segment plasma membrane and disk-disk stacking (Kaupp \& Seifert 2002). These findings,

482 together with the lack of outer segment disk pathology in pigmented $\mathrm{Abca4}^{-/}$mice (Table 1)

483 (Charbel Issa et al. 2013), indicate that ABCA4 is not needed for proper disk formation,

484 orientation and stability. Whether RPE malfunction is responsible for the presented outer

485 segment defects in albino $\mathrm{Abca}^{-/-}$and to a lesser extent $\mathrm{Abca4^{-/ }}$. $\mathrm{Rdh}^{-/-}$mice needs to be 486 elucidated.

487

488

4.2 Indications for lysosomal dysfunction and oxidative stress in albino $\mathrm{Abca}^{-/-}$mice

489 Formation of vacuole-like structures in the RPE as seen in albino $\mathrm{Abca} 4^{-/}$mice (Fig. 3) has been

490 described after lysosomal integrity has been compromised and after oxidative stress. Increasing

491 the lysosomal $\mathrm{pH}$ with chloroquine (Peters et al. 2006), inhibiting lysosomal cysteine proteases

492 (Okubo et al. 2000) and a lack of functional $\beta$ A3/A1-crystallin (Valapala et al. 2014; Zigler et al. 
493 2011) all lead to formation of vacuole-like structures within the RPE cell layer. Lysosomal pH 494 was shown to be increased after treatment of RPE cells with the lipofuscin compound A2E in 495 vitro (Holz et al. 1999; Liu et al. 2008) and in primary RPE cells isolated from pigmented Abca4 496 /- mice (Liu et al. 2008). Since the melanosome is a specialized lysosome (Orlow 1995) that 497 contains many lysosomal enzymes (Diment et al. 1995) and can fuse with phagosomes 498 (Schraermeyer et al. 1999), it is possible that albinism itself adds to the lysosomal dysfunction 499 seen in albino $\mathrm{Abca4}^{-/}$mice due to lack of melanosomes.

500 Similar pathologies were also described after oxidative stress. Changes in RPE morphology, such 501 as vacuolization and loss of basal labyrinth, and deposit formation were seen after a lack of 502 superoxide dismutase (Mao et al. 2014; Seo et al. 2012), knockout of Nrf2 , a transcription factor 503 that regulates genes involved in the cellular oxidative stress response (Zhao et al. 2011), and 504 after chronic exposure to cigarette smoke (Fujihara et al. 2008). In some models, thinning of the 505 outer nuclear layer (Mao et al. 2014; Seo et al. 2012) and shortening and disorganization of inner 506 and outer segments (Mao et al. 2014) or RPE hyper- and hypopigmentation and choroidal 507 neovascularization (Zhao et al. 2011) were also described. Besides the high oxygen partial 508 pressure and the presence of light, other sources for oxidative stress in the RPE are phagocytosis 509 of photoreceptor outer segments (Miceli et al. 1994) and lipofuscin (Rozanowska et al. 1995). 510 Previous studies investigating oxidative stress in Stargardt mice found that albino $A b c a 4^{-/}$mice 511 have higher levels of MDA and HNE adducts than age-matched albino WT and pigmented $512 \mathrm{Abca}^{4 /-}$ mice (Radu et al. 2011) and that 6-month-old $\mathrm{Abca4^{-/ }}$. Rdh $8^{-/ /}$mice have higher levels of 513 8-OHdG than 4-week-old $A b c a 4^{--} \cdot R d h 8^{-/-}$mice and age-matched WT mice (Sawada et al. 2014).

514 We found similar results indicating that pigmented $\mathrm{Abca}^{-/}$mice have the lowest oxidative stress 515 levels (Fig. 9). 
516 Interestingly, $\mathrm{Abca}^{-/} . \mathrm{Rdh} 8^{-/}$mice had higher numbers of undegraded phagocytosed outer 517 segments than albino Abca4 $4^{-/}$mice (Table 1), but albino $\mathrm{Abca}^{-/}$mice had higher numbers of

518 vacuole-like structures (Fig. 3 D). One reason might be higher oxidative stress levels in albino

519 Abca4 $4^{-/}$mice. Melanin was shown to be a scavenger of superoxide anion and singlet oxygen

520 (Bustamante et al. 1993; Tada et al. 2010) and protects human RPE cells from oxidative stress in

521 vitro (Burke et al. 2011). Intravitreal application of paraquat, a superoxide anion generator, leads

522 to higher superoxide levels and more pronounced lipid oxidation and retinal degeneration in

523 albino compared to pigmented mice (Cingolani et al. 2006).

524 Additionally, changes of RPE morphology in albino animals might play a role in RPE

525 vacuolization: embryonic RPE from albino mice was found to have an irregular morphology,

526 compared to pigmented littermates (Iwai-Takekoshi et al. 2016). Furthermore, an altered

527 distribution of the junctional protein P-cadherin was found in albino RPE and it was

528 hypothesized that this might lead to defects in cell-cell adhesion (Iwai-Takekoshi et al. 2016). It

529 is reasonable that a combination of weakened cell-cell contacts, impaired lysosomal function and

530 elevated oxidative stress as described above might lead to the defects found in albino $A b c a 4^{-/}$

531 mice.

532

533

4.3 Lipofuscin varies considerably between Stargardt mouse strains

534 Morphological appearance of lipofuscin granules differs in the three different Stargardt mouse

535 strains. It has been described that the chemical composition of lipofuscin in pigmented and

536 albino WT mice differs in terms of the amounts of the different types of bisretinoids that can be

537 found (Ueda et al. 2016). It is hypothesized that the higher intraocular light levels in albino

538 animals lead to photooxidation and photodegradation of A2E and other bisretinoids (Ueda et al. 
539 2016), which is in accordance with earlier publications that demonstrated lower A2E and higher

540 A2E oxirane levels in albino $\mathrm{Abca4^{-/ }}$ mice compared to pigmented $\mathrm{Abca4^{-/ }}$ mice (Radu et al.

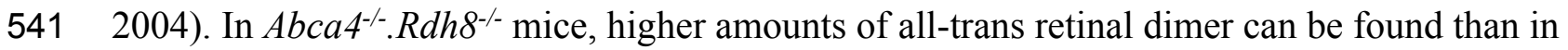

$542 \mathrm{Abca}^{-/ /}$mice (Maeda et al. 2008). Our results show that in addition to the difference in the

543 chemical composition of lipofuscin between the three strains, there is also a considerable

544 histological difference.

545 Strikingly, lipofuscin levels in 12-month-old animals seem to be elevated in albino $\mathrm{Abca4}^{-/}$

546 compared to pigmented $\mathrm{Abca4} 4^{-/}$mice, when investigated by lipofuscin autofluorescence (Fig. 4

547 A), while quantification of RPE cytoplasm occupied by lipofuscin granules shows similar

548 lipofuscin levels in 12-month-old pigmented and albino Abca4-- mice (Fig. 5). Both quenching

549 effects of the melanin and differences in nature and amount of present fluorophores, as described 550 above, are possible explanations for this seeming contradiction.

551 The Met450 variant of RPE65 was described to be associated with lower levels of the lipofuscin

552 fluorophores A2E and iso-A2E in $\mathrm{Abca}^{-/} . \mathrm{Rdh}^{-/-}$mice (Kim et al. 2004). Since pigmented and

553 albino $\mathrm{Abca} 4^{-/}$mice carry the Leu450 variant of RPE65 and we did not genotype the $\mathrm{Abca4^{-/ }}$

$554 . R d h 8^{-/-}$mice for RPE65, lipofuscin levels in Figs. 4 and 5 in $A b c a 4^{-/} \cdot R d h 8^{-/-}$mice might be 555 underreported.

556 The bisretinoid A2E was shown to have surfactant-like properties and to be able to compromise 557 lysosomal and cellular membranes (Schutt et al. 2002; Sparrow et al. 1999). In fact, we found 558 that lipofuscin granules often lacked membranes (Fig. 4 C). Furthermore, in very old animals

559 (aged 23 months and older), we found abnormally electron dense RPE cytoplasm (Fig. 4 D), 560 making distinguishing between lipofuscin granules and cytoplasm very difficult. Whether the 
561 RPE cytoplasm gained electron density due to free lipofuscin components that leaked from

562 lipofuscin granules after losing their membranes is unclear but appears likely.

563

564

4.4 Bruch's membrane thickness does not vary between Stargardt mouse strains

565 For all three Stargardt mouse strains, a thickening of Bruch's membrane compared to WT was

566 described (Maeda et al. 2008; Radu et al. 2011; Weng et al. 1999). Compared to each other, the

567 three strains show comparable thickening of Bruch's membrane with age (Fig. 7). An increase in

568 the thickness of Bruch's membrane is also typical in Stargardt patients (Bonilha et al. 2016).

569

570

4.5 The significance of RPE melanin and its role as a scavenger of oxidative stress

571 The role of reactive oxygen species in retinal health was extensively investigated (Becquet et al.

572 1994; Cingolani et al. 2006; Mao et al. 2014; Seo et al. 2012; Zhao et al. 2011) and melanin was

573 identified as a potential scavenger of reactive oxygen species (Bustamante et al. 1993; Tada et al.

574 2010). However, RPE melanin differs from other melanin species in several aspects. For

575 instance, while melanocytes continuously synthesize new melanin, it is still under debate

576 whether melanin turnover in the RPE occurs and if so, to what extent (Schraermeyer 1993).

577 Furthermore, RPE melanin can react differently than melanin in the melanocytes of the choroid

578 (Schraermeyer \& Heimann 1999). Therefore, we analyzed isolated RPE melanin granules to

579 corroborate that also this melanin species is capable to quench superoxide radicals and in fact

580 can play a role in protection against oxidative stress in the RPE (Fig. 10). Anti-oxidative

581 properties of RPE melanin have also been confirmed in cell culture experiments (Burke et al.

582 2011). 
583 Certain photoreceptor and RPE-damages typical for oxidative stress and/or lysosomal

584 impairment were exclusively found in albino $\mathrm{Abca}^{-/-}$mice (Figures $1 \mathrm{D}, 2 \mathrm{~B}-\mathrm{D}, 3$ and $6 \mathrm{C}$ ),

585 while others were found in albino $\mathrm{Abca}^{-/-}$and $\mathrm{Abca4^{-/ }} \cdot \mathrm{R} d \mathrm{~h} 8^{-/-}$mice, but onset was earlier in

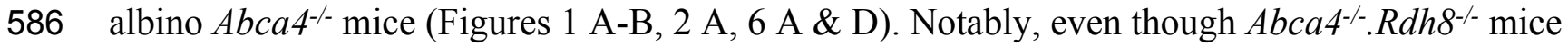

587 have a more severe disease-causing genotype compared to albino $\mathrm{Abca}^{-/-}$mice, they only show

588 a more pathologic phenotype regarding RPE hypertrophy (Fig. 1 A, Fig. S1), the number of

589 undegraded phagosomes (Table 1) and the percentage of RPE cytoplasm occupied by lipofuscin 590 (Fig. 5).

591

592

4.5.1 RPE melanin affects the accumulation of lipofuscin, a major hallmark of Stargardt

593 disease

594 Melanin in the RPE plays a crucial role as scavenger for excess light and reactive oxygen species

595 (Hu et al. 2008). Lack of melanin has been associated with higher levels of lipofuscin in primary 596 RPE cells in vitro (Sundelin et al. 2001) and a decrease of A2E with simultaneous increase of

597 A2E oxiranes in albino compared to pigmented $\mathrm{Abca}^{4^{-/-}}$mice (Radu et al. 2004). We show that

598 albino $\mathrm{Abca4}^{-/-}$mice have higher levels of lipofuscin than pigmented $\mathrm{Abca4}^{-/}$mice (Fig. 5).

599 We identified different morphologies of lipofuscin in the three different Stargardt mouse strains.

600 In young age, pigmented $\mathrm{Abca}^{-/-}$and $\mathrm{Abca}^{-/-} \cdot \mathrm{Rdh}^{-/-}$mice show exclusively lipofuscin with

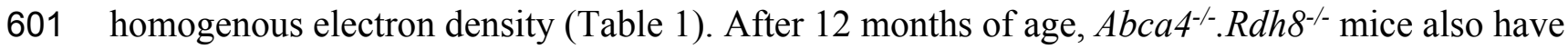

602 lipofuscin with flocculent electron density. Contrary, albino $\mathrm{Abca}^{-/-}$mice have little lipofuscin

603 with homogenous electron density, while lipofuscin with flocculent electron density is present

604 even in young animals (Table 1).

605 
$606 \quad$ 4.5.2 Aged melanin becomes pro-oxidative and might be involved in Stargardt pathology 607 Melanin granules probably play an essential role in the detoxification of lipofuscin due to their 608 anti-oxidative properties (Burke et al. 2011). With age, melanin granules switch from expressing 609 anti-oxidative to pro-oxidative properties after illumination (Biesemeier et al. 2008; Dontsov et 610 al. 1999; Rozanowski et al. 2008). This might be due to degradative modifications of melanin 611 that occur after long-term interaction of iron-containing melanin with lipid hydroperoxides, as it 612 happens in melanolipofuscin (Zadlo et al. 2017). Nevertheless, formation of melanolipofuscin 613 might be a protective process, since radicals formed by degradation of bisretinoids (Wu et al. 614 2011) can be absorbed by the melanin moiety of melanolipofuscin and thereby protect the 615 lysosomal membrane from damage.

616 In fact, alterations in near-infrared-autofluorescence, which is a marker for melanin, were 617 identified as the earliest detectable retinal change in patients with $A B C A 4$-related retinal 618 dystrophies and can be used for predicting the further disease progress (Cideciyan et al. 2015). 619 Changes in near-infrared-autofluorescence were also found in pigmented $A b c a 4^{-/}$mice 620 compared to wild type (Charbel Issa et al. 2013). Since $A b c a 4^{--} \cdot$ Rdh $8^{-/-}$mice have higher loads 621 of the pro-oxidative substances lipofuscin (Rozanowska et al. 1995) and all-trans-retinal (Zhu et 622 al. 2016) than pigmented $A b c a 4^{-/}$mice, their melanin granules might age more rapidly and 623 switch to the pro-oxidative state earlier. This might explain the presence of lipofuscin with 624 flocculent electron density in aged $\mathrm{Abca}^{-/} \cdot \mathrm{Rdh}^{-/}$mice.

625

626

4.5.3 RPE melanin and its link to outer segment phagocytosis and ingestion of bisretinoids

627 Phagocytosis of outer segments was shown to be an oxidative stressor for the RPE (Miceli et al.

628 1994). Since undegradable material from phagocytosed outer segments is transported to melanin 
629 granules in primary RPE cells (Schraermeyer et al. 1999; Schraermeyer \& Stieve 1994) and

630 melanosomes can bind and store many toxic components (Mecklenburg \& Schraermeyer 2007),

631 melanin granules might play a role in lowering phagocytosis-related oxidative or toxic stress.

632 When post-confluent differentiated ARPE-19 cells are treated with low micromolar amounts of

633 A2E for several weeks and then challenged with outer segments, melanin synthesis is induced

634 (Poliakov et al. 2014). Poliakov et al. even proposed that A2E-induced alkalization of lysosomes

635 could have a physiological role in maintaining melanin pigmentation in RPE cells, since neutral

$636 \mathrm{pH}$ favors eumelanogenesis (Fuller et al. 2001; Ito et al. 2013).

637

$638 \quad$ 4.5.4 There is still much to be learned

639 Taken together, all these observations point to RPE melanin being an essential factor for retinal 640 function. Complete lack of RPE melanin granules in albino $\mathrm{Abca4}^{-/-}$mice leads to extensive

641 retinal damage that cannot be found in pigmented tissue, even in $A b c a 4^{--} \cdot R d h 8^{-/-}$mice that have

642 an increased amount of toxic visual cycle byproducts like all-trans retinal dimer and higher levels

643 of lipofuscin due to their genetic burden. We cannot rule out that the lack of melanin in the iris

644 and the choroid is an additional reinforcing factor in albino animals, but there is overwhelming

645 data supporting the essential role of RPE melanin. Furthermore, yet unidentified differences in

646 the genetic background of the different mouse strains might skew the presented data.

647 Ocular pigmentation was shown to be correlated to the incidence of uveal melanoma (Weis et al.

648 2006) and age-related macular degeneration (Chakravarthy et al. 2010) and tyrosinase, the key

649 enzyme for melanin biogenesis, was found to be a potential factor for developing early stages of

650 age-related macular degeneration (Holliday et al. 2013). 


\section{Conclusion}

652 In this work, we present a detailed ultrastructural comparison of the disease development of three

653 frequently used Stargardt mouse models. As currently no animal model exists that reflects all

654 aspects of the human disease, this will help researchers in the process of identifying which model 655 is best suited for their research.

656 We found that albino $\mathrm{Abca}^{-/-}$and $\mathrm{Abca}^{-/-} \cdot \mathrm{R}_{\mathrm{Ch}} 8^{-/-}$mice show typical histopathological signs of 657 oxidative stress and/or lysosomal dysfunction, the earliest onset and severest changes being 658 present in albino $\mathrm{Abca4^{-- }}$ mice. Since melanin in general is known to have anti-oxidative 659 properties and age-dependent turnover of melanin is known to diminish the anti-oxidative 660 properties of the RPE, we hypothesize that RPE melanin plays a crucial role in preventing 661 Stargardt-related changes. The lack of pathology in pigmented $\mathrm{Abca}^{-/-}$mice and the finding that 662 also RPE melanin can quench superoxide anions support this hypothesis.

663 The protective role of melanin due to its antioxidative properties might be an explanation for the 664 relatively late-onset of Stargardt disease with mild to moderate disease causing mutations (van 665 Huet et al. 2014): as long as melanin is capable of alleviating toxic effects of lipofuscin 666 accumulation, RPE health is maintained. Once melanin is aged and lost its protective function, 667 deleterious effects of lipofuscin accumulation can take over. This is strongly supported by the 668 fact that changes in melanin-based near-infrared-autofluorescence temporally and spatially 669 precede the progression of retinal degeneration in Stargardt disease (Cideciyan et al. 2015).

670 Since RPE damage is a fundamental feature of Stargardt disease and age-related macular 671 degeneration, RPE melanin should be strongly considered as a key factor in retinal health. 


\section{Acknowledgements}

673 The authors thank Peter Charbel Issa, Gabriel H. Travis, Roxana Radu and Krzysztof Palczewski

674 for providing them with the Stargardt mouse strains. Special thanks are due to Foundation

675 Fighting Blindness, Vera Bonilha and Joe Hollyfield for collecting and providing the human

676 Stargardt tissue. In this work, the segmentation calculations were performed using Fiji on the

677 de.NBI Cloud Tübingen (https://denbi.uni-tuebingen.de). The authors thank Jens Krüger,

678 Maximilian Hanussek and Felix Bartusch of the High Performance and Cloud Computing Group

679 at the Zentrum für Datenverarbeitung of the University of Tuebingen. The authors also thank

680 Monika Rittgarn and Sigrid Schultheiss for technical assistance and Daniel Dehncke for helpful

681 discussions. 
682

683

684

685

686

687

688

689

690

691

692

693

694

695

696

697

698

699

700

701

702

703

704

705

706

707

708

709

710

711

712

713

714

715

716

717

718

719

720

721

722

723

724

725

726

727

728

729

\section{References}

Allikmets R, Singh N, Sun H, Shroyer NF, Hutchinson A, Chidambaram A, Gerrard B, Baird L, Stauffer D, Peiffer A, Rattner A, Smallwood P, Li Y, Anderson KL, Lewis RA, Nathans J, Leppert M, Dean M, and Lupski JR. 1997. A photoreceptor cell-specific ATP-binding transporter gene (ABCR) is mutated in recessive Stargardt macular dystrophy. Nat Genet 15:236-246. 10.1038/ng0397-236

Arganda-Carreras I, Kaynig V, Rueden C, Eliceiri KW, Schindelin J, Cardona A, and Sebastian Seung H. 2017. Trainable Weka Segmentation: a machine learning tool for microscopy pixel classification. Bioinformatics 33:2424-2426. 10.1093/bioinformatics/btx180

Becquet F, Goureau O, Soubrane G, Coscas G, Courtois Y, and Hicks D. 1994. Superoxide inhibits proliferation and phagocytic internalization of photoreceptor outer segments by bovine retinal pigment epithelium in vitro. Exp Cell Res 212:374-382. 10.1006/excr.1994.1157

Ben-Shabat S, Itagaki Y, Jockusch S, Sparrow JR, Turro NJ, and Nakanishi K. 2002. Formation of a nonaoxirane from $\mathrm{A} 2 \mathrm{E}$, a lipofuscin fluorophore related to macular degeneration, and evidence of singlet oxygen involvement. Angew Chem Int Ed Engl 41:814-817.

Biesemeier A, Kokkinou D, Julien S, Heiduschka P, Berneburg M, Bartz-Schmidt KU, and Schraermeyer U. 2008. UV-A induced oxidative stress is more prominent in naturally pigmented aged human RPE cells compared to non-pigmented human RPE cells independent of zinc treatment. J Photochem Photobiol B 90:113-120. 10.1016/j.jphotobiol.2007.11.005

Birnbach CD, Jarvelainen M, Possin DE, and Milam AH. 1994. Histopathology and immunocytochemistry of the neurosensory retina in fundus flavimaculatus. Ophthalmology 101:1211-1219.

Blacharski P. 1988. Fundus flavimaculatus. In: Newsome DA, ed. Retinal Dystrophies and Degenerations New York: Raven Press, 135-159.

Bonilha VL, Rayborn ME, Bell BA, Marino MJ, Fishman GA, and Hollyfield JG. 2016. Retinal Histopathology in Eyes from a Patient with Stargardt disease caused by Compound Heterozygous ABCA4 Mutations. Ophthalmic Genet 37:150-160. 10.3109/13816810.2014.958861

Boulton M, and Marshall J. 1985. Repigmentation of human retinal pigment epithelial cells in vitro. Exp Eye Res 41:209-218.

Burke JM, Kaczara P, Skumatz CM, Zareba M, Raciti MW, and Sarna T. 2011. Dynamic analyses reveal cytoprotection by RPE melanosomes against non-photic stress. Mol Vis 17:2864-2877.

Bustamante J, Bredeston L, Malanga G, and Mordoh J. 1993. Role of melanin as a scavenger of active oxygen species. Pigment Cell Res 6:348-353.

Chakravarthy U, Wong TY, Fletcher A, Piault E, Evans C, Zlateva G, Buggage R, Pleil A, and Mitchell P. 2010. Clinical risk factors for age-related macular degeneration: a systematic review and meta-analysis. BMC Ophthalmol 10:31. 10.1186/1471-2415-10-31

Charbel Issa P, Barnard AR, Singh MS, Carter E, Jiang Z, Radu RA, Schraermeyer U, and MacLaren RE. 2013. Fundus autofluorescence in the Abca4(-/-) mouse model of Stargardt disease--correlation with accumulation of A2E, retinal function, and histology. Invest Ophthalmol Vis Sci 54:5602-5612. 10.1167/iovs.13-11688

Cheng CW, Chen LY, Chou CW, and Liang JY. 2015. Investigations of riboflavin photolysis via coloured light in the nitro blue tetrazolium assay for superoxide dismutase activity. $J$ Photochem Photobiol B 148:262-267. 10.1016/j.jphotobiol.2015.04.028 
730

731

732

733

734

735

736

737

738

739

740

741

742

743

744

745

746

747

748

749

750

751

752

753

754

755

756

757

758

759

760

761

762

763

764

765

766

767

768

769

770

771

772

773

774

775

776

777

778

779

Cideciyan AV, Swider M, Schwartz SB, Stone EM, and Jacobson SG. 2015. Predicting Progression of ABCA4-Associated Retinal Degenerations Based on Longitudinal Measurements of the Leading Disease Front. Invest Ophthalmol Vis Sci 56:5946-5955. 10.1167/iovs.15-17698

Cingolani C, Rogers B, Lu L, Kachi S, Shen J, and Campochiaro PA. 2006. Retinal degeneration from oxidative damage. Free Radic Biol Med 40:660-669. 10.1016/j.freeradbiomed.2005.09.032

Danciger M, Lyon J, Worrill D, Hoffman S, Lem J, Reme CE, Wenzel A, and Grimm C. 2004. New retinal light damage QTL in mice with the light-sensitive RPE65 LEU variant. Mamm Genome 15:277-283. 10.1007/s00335-003-2336-2

Diment S, Eidelman M, Rodriguez GM, and Orlow SJ. 1995. Lysosomal hydrolases are present in melanosomes and are elevated in melanizing cells. J Biol Chem 270:4213-4215.

Dontsov AE, Glickman RD, and Ostrovsky MA. 1999. Retinal pigment epithelium pigment granules stimulate the photo-oxidation of unsaturated fatty acids. Free Radic Biol Med 26:1436-1446.

Eagle RC, Jr., Lucier AC, Bernardino VB, Jr., and Yanoff M. 1980. Retinal pigment epithelial abnormalities in fundus flavimaculatus: a light and electron microscopic study. Ophthalmology 87:1189-1200.

Fujihara M, Nagai N, Sussan TE, Biswal S, and Handa JT. 2008. Chronic cigarette smoke causes oxidative damage and apoptosis to retinal pigmented epithelial cells in mice. PLoS One 3:e3119. 10.1371/journal.pone.0003119

Fuller BB, Spaulding DT, and Smith DR. 2001. Regulation of the catalytic activity of preexisting tyrosinase in black and Caucasian human melanocyte cell cultures. Exp Cell Res 262:197-208. 10.1006/excr.2000.5092

Goldberg AF, Moritz OL, and Williams DS. 2016. Molecular basis for photoreceptor outer segment architecture. Prog Retin Eye Res 55:52-81. 10.1016/j.preteyeres.2016.05.003

Gomes NL, Greenstein VC, Carlson JN, Tsang SH, Smith RT, Carr RE, Hood DC, and Chang S. 2009. A comparison of fundus autofluorescence and retinal structure in patients with Stargardt disease. Invest Ophthalmol Vis Sci 50:3953-3959. 10.1167/iovs.08-2657

Holliday EG, Smith AV, Cornes BK, Buitendijk GH, Jensen RA, Sim X, Aspelund T, Aung T, Baird PN, Boerwinkle E, Cheng CY, van Duijn CM, Eiriksdottir G, Gudnason V, Harris T, Hewitt AW, Inouye M, Jonasson F, Klein BE, Launer L, Li X, Liew G, Lumley T, McElduff P, McKnight B, Mitchell P, Psaty BM, Rochtchina E, Rotter JI, Scott RJ, Tay W, Taylor K, Teo YY, Uitterlinden AG, Viswanathan A, Xie S, Wellcome Trust Case Control C, Vingerling JR, Klaver CC, Tai ES, Siscovick D, Klein R, Cotch MF, Wong TY, Attia J, and Wang JJ. 2013. Insights into the genetic architecture of early stage age-related macular degeneration: a genome-wide association study meta-analysis. PLoS One 8:e53830. 10.1371/journal.pone.0053830

Holz FG, Schutt F, Kopitz J, Eldred GE, Kruse FE, Volcker HE, and Cantz M. 1999. Inhibition of lysosomal degradative functions in RPE cells by a retinoid component of lipofuscin. Invest Ophthalmol Vis Sci 40:737-743.

Hu DN, Simon JD, and Sarna T. 2008. Role of ocular melanin in ophthalmic physiology and pathology. Photochem Photobiol 84:639-644. 10.1111/j.1751-1097.2008.00316.x

Huttl S, Michalakis S, Seeliger M, Luo DG, Acar N, Geiger H, Hudl K, Mader R, Haverkamp S, Moser M, Pfeifer A, Gerstner A, Yau KW, and Biel M. 2005. Impaired channel targeting and retinal degeneration in mice lacking the cyclic nucleotide-gated channel subunit CNGB1. J Neurosci 25:130-138. 10.1523/JNEUROSCI.3764-04.2005

Ito S, Suzuki N, Takebayashi S, Commo S, and Wakamatsu K. 2013. Neutral pH and copper ions promote eumelanogenesis after the dopachrome stage. Pigment Cell Melanoma Res 26:817-825. 10.1111/pcmr.12137 
780

781

782

783

784

785

786

787

788

789

790

791

792

793

794

795

796

797

798

799

800

801

802

803

804

805

806

807

808

809

810

811

812

813

814

815

816

817

818

819

820

821

822

823

824

825

826

827

828

829

Iwai-Takekoshi L, Ramos A, Schaler A, Weinreb S, Blazeski R, and Mason C. 2016. Retinal pigment epithelial integrity is compromised in the developing albino mouse retina. $J$ Comp Neurol 524:3696-3716. 10.1002/cne.24025

Kaupp UB, and Seifert R. 2002. Cyclic nucleotide-gated ion channels. Physiol Rev 82:769-824. 10.1152/physrev.00008.2002

Kim SR, Fishkin N, Kong J, Nakanishi K, Allikmets R, and Sparrow JR. 2004. Rpe65 Leu450Met variant is associated with reduced levels of the retinal pigment epithelium lipofuscin fluorophores A2E and iso-A2E. Proc Natl Acad Sci U S A 101:11668-11672. 10.1073/pnas.0403499101

Lem J, Krasnoperova NV, Calvert PD, Kosaras B, Cameron DA, Nicolo M, Makino CL, and Sidman RL. 1999. Morphological, physiological, and biochemical changes in rhodopsin knockout mice. Proc Natl Acad Sci U S A 96:736-741.

Liu J, Lu W, Reigada D, Nguyen J, Laties AM, and Mitchell CH. 2008. Restoration of lysosomal $\mathrm{pH}$ in RPE cells from cultured human and ABCA4(-/-) mice: pharmacologic approaches and functional recovery. Invest Ophthalmol Vis Sci 49:772-780. 10.1167/iovs.07-0675

Maeda A, Maeda T, Golczak M, and Palczewski K. 2008. Retinopathy in mice induced by disrupted all-trans-retinal clearance. J Biol Chem 283:26684-26693. 10.1074/jbc.M804505200

Mao H, Seo SJ, Biswal MR, Li H, Conners M, Nandyala A, Jones K, Le YZ, and Lewin AS. 2014. Mitochondrial oxidative stress in the retinal pigment epithelium leads to localized retinal degeneration. Invest Ophthalmol Vis Sci 55:4613-4627. 10.1167/iovs.14-14633

Mecklenburg L, and Schraermeyer U. 2007. An overview on the toxic morphological changes in the retinal pigment epithelium after systemic compound administration. Toxicol Pathol 35:252-267. 10.1080/01926230601178199

Miceli MV, Liles MR, and Newsome DA. 1994. Evaluation of oxidative processes in human pigment epithelial cells associated with retinal outer segment phagocytosis. Exp Cell Res 214:242-249. 10.1006/excr.1994.1254

Molday RS, Zhong M, and Quazi F. 2009. The role of the photoreceptor ABC transporter ABCA4 in lipid transport and Stargardt macular degeneration. Biochim Biophys Acta 1791:573-583. 10.1016/j.bbalip.2009.02.004

Okubo A, Sameshima M, Unoki K, Uehara F, and Bird AC. 2000. Ultrastructural changes associated with accumulation of inclusion bodies in rat retinal pigment epithelium. Invest Ophthalmol Vis Sci 41:4305-4312.

Orlow SJ. 1995. Melanosomes are specialized members of the lysosomal lineage of organelles. J Invest Dermatol 105:3-7.

Papermaster DS, Schneider BG, Zorn MA, and Kraehenbuhl JP. 1978. Immunocytochemical localization of a large intrinsic membrane protein to the incisures and margins of frog rod outer segment disks. J Cell Biol 78:415-425.

Peters S, Reinthal E, Blitgen-Heinecke P, Bartz-Schmidt KU, and Schraermeyer U. 2006. Inhibition of lysosomal degradation in retinal pigment epithelium cells induces exocytosis of phagocytic residual material at the basolateral plasma membrane. Ophthalmic Res 38:83-88. 10.1159/000090268

Poetsch A, Molday LL, and Molday RS. 2001. The cGMP-gated channel and related glutamic acid-rich proteins interact with peripherin-2 at the rim region of rod photoreceptor disc membranes. J Biol Chem 276:48009-48016. 10.1074/jbc.M108941200

Poliakov E, Strunnikova NV, Jiang JK, Martinez B, Parikh T, Lakkaraju A, Thomas C, Brooks BP, and Redmond TM. 2014. Multiple A2E treatments lead to melanization of rod outer segment-challenged ARPE-19 cells. Mol Vis 20:285-300.

Radu RA, Hu J, Yuan Q, Welch DL, Makshanoff J, Lloyd M, McMullen S, Travis GH, and Bok D. 2011. Complement system dysregulation and inflammation in the retinal pigment 
830

831

832

833

834

835

836

837

838

839

840

841

842

843

844

845

846

847

848

849

850

851

852

853

854

855

856

857

858

859

860

861

862

863

864

865

866

867

868

869

870

871

872

873

874

875

876

877

878

879 epithelium of a mouse model for Stargardt macular degeneration. J Biol Chem 286:18593-18601. 10.1074/jbc.M110.191866

Radu RA, Mata NL, Bagla A, and Travis GH. 2004. Light exposure stimulates formation of A2E oxiranes in a mouse model of Stargardt's macular degeneration. Proc Natl Acad Sci U S A 101:5928-5933. 10.1073/pnas.0308302101

Radu RA, Yuan Q, Hu J, Peng JH, Lloyd M, Nusinowitz S, Bok D, and Travis GH. 2008. Accelerated accumulation of lipofuscin pigments in the RPE of a mouse model for ABCA4-mediated retinal dystrophies following Vitamin A supplementation. Invest Ophthalmol Vis Sci 49:3821-3829. 10.1167/iovs.07-1470

Rozanowska M, Jarvis-Evans J, Korytowski W, Boulton ME, Burke JM, and Sarna T. 1995. Blue light-induced reactivity of retinal age pigment. In vitro generation of oxygen-reactive species. J Biol Chem 270:18825-18830.

Rozanowski B, Cuenco J, Davies S, Shamsi FA, Zadlo A, Dayhaw-Barker P, Rozanowska M, Sarna T, and Boulton ME. 2008. The phototoxicity of aged human retinal melanosomes. Photochem Photobiol 84:650-657. 10.1111/j.1751-1097.2007.00259.x

Sawada O, Perusek L, Kohno H, Howell SJ, Maeda A, Matsuyama S, and Maeda T. 2014. Alltrans-retinal induces Bax activation via DNA damage to mediate retinal cell apoptosis. Exp Eye Res 123:27-36. 10.1016/j.exer.2014.04.003

Schindelin J, Arganda-Carreras I, Frise E, Kaynig V, Longair M, Pietzsch T, Preibisch S, Rueden C, Saalfeld S, Schmid B, Tinevez JY, White DJ, Hartenstein V, Eliceiri K, Tomancak P, and Cardona A. 2012. Fiji: an open-source platform for biological-image analysis. Nat Methods 9:676-682. 10.1038/nmeth.2019

Schraermeyer U. 1993. Does melanin turnover occur in the eyes of adult vertebrates? Pigment Cell Res 6:193-204.

Schraermeyer U, and Heimann K. 1999. Current understanding on the role of retinal pigment epithelium and its pigmentation. Pigment Cell Res 12:219-236.

Schraermeyer U, Peters S, Thumann G, Kociok N, and Heimann K. 1999. Melanin granules of retinal pigment epithelium are connected with the lysosomal degradation pathway. Exp Eye Res 68:237-245. 10.1006/exer.1998.0596

Schraermeyer U, and Stieve H. 1994. A newly discovered pathway of melanin formation in cultured retinal pigment epithelium of cattle. Cell Tissue Res 276:273-279.

Schutt F, Bergmann M, Holz FG, and Kopitz J. 2002. Isolation of intact lysosomes from human RPE cells and effects of A2-E on the integrity of the lysosomal and other cellular membranes. Graefes Arch Clin Exp Ophthalmol 240:983-988. 10.1007/s00417-0020558-8

Seo SJ, Krebs MP, Mao H, Jones K, Conners M, and Lewin AS. 2012. Pathological consequences of long-term mitochondrial oxidative stress in the mouse retinal pigment epithelium. Exp Eye Res 101:60-71. 10.1016/j.exer.2012.05.013

Sparrow JR, Blonska A, Flynn E, Duncker T, Greenberg JP, Secondi R, Ueda K, and Delori FC. 2013. Quantitative fundus autofluorescence in mice: correlation with HPLC quantitation of RPE lipofuscin and measurement of retina outer nuclear layer thickness. Invest Ophthalmol Vis Sci 54:2812-2820. 10.1167/iovs.12-11490

Sparrow JR, Gregory-Roberts E, Yamamoto K, Blonska A, Ghosh SK, Ueda K, and Zhou J. 2012. The bisretinoids of retinal pigment epithelium. Prog Retin Eye Res 31:121-135. 10.1016/j.preteyeres.2011.12.001

Sparrow JR, Parish CA, Hashimoto M, and Nakanishi K. 1999. A2E, a lipofuscin fluorophore, in human retinal pigmented epithelial cells in culture. Invest Ophthalmol Vis Sci 40:29882995.

Sparrow JR, Vollmer-Snarr HR, Zhou J, Jang YP, Jockusch S, Itagaki Y, and Nakanishi K. 2003a. A2E-epoxides damage DNA in retinal pigment epithelial cells. Vitamin E and 
880

881

882

883

884

885

886

887

888

889

890

891

892

893

894

895

896

897

898

899

900

901

902

903

904

905

906

907

908

909

910

911

912

913

914

915

916

917

918

919

920

921

922

923

924

925

926

927

928

929

other antioxidants inhibit A2E-epoxide formation. J Biol Chem 278:18207-18213. 10.1074/jbc.M300457200

Sparrow JR, Zhou J, and Cai B. 2003b. DNA is a target of the photodynamic effects elicited in A2E-laden RPE by blue-light illumination. Invest Ophthalmol Vis Sci 44:2245-2251.

Sundelin SP, Nilsson SE, and Brunk UT. 2001. Lipofuscin-formation in cultured retinal pigment epithelial cells is related to their melanin content. Free Radic Biol Med 30:74-81.

Tada M, Kohno M, and Niwano Y. 2010. Scavenging or quenching effect of melanin on superoxide anion and singlet oxygen. J Clin Biochem Nutr 46:224-228. 10.3164/jcbn.0984

Ueda K, Zhao J, Kim HJ, and Sparrow JR. 2016. Photodegradation of retinal bisretinoids in mouse models and implications for macular degeneration. Proc Natl Acad Sci U S A 113:6904-6909. 10.1073/pnas.1524774113

Valapala M, Wilson C, Hose S, Bhutto IA, Grebe R, Dong A, Greenbaum S, Gu L, Sengupta S, Cano M, Hackett S, Xu G, Lutty GA, Dong L, Sergeev Y, Handa JT, Campochiaro P, Wawrousek E, Zigler JS, Jr., and Sinha D. 2014. Lysosomal-mediated waste clearance in retinal pigment epithelial cells is regulated by CRYBA1/betaA3/A1-crystallin via VATPase-MTORC1 signaling. Autophagy 10:480-496. 10.4161/auto.27292

van Huet RA, Bax NM, Westeneng-Van Haaften SC, Muhamad M, Zonneveld-Vrieling MN, Hoefsloot LH, Cremers FP, Boon CJ, Klevering BJ, and Hoyng CB. 2014. Foveal sparing in Stargardt disease. Invest Ophthalmol Vis Sci 55:7467-7478. 10.1167/iovs.13-13825

Weis E, Shah CP, Lajous M, Shields JA, and Shields CL. 2006. The association between host susceptibility factors and uveal melanoma: a meta-analysis. Arch Ophthalmol 124:54-60. 10.1001/archopht.124.1.54

Weng J, Mata NL, Azarian SM, Tzekov RT, Birch DG, and Travis GH. 1999. Insights into the function of Rim protein in photoreceptors and etiology of Stargardt's disease from the phenotype in abcr knockout mice. Cell 98:13-23. 10.1016/S0092-8674(00)80602-9

Wu L, Nagasaki T, and Sparrow JR. 2010. Photoreceptor cell degeneration in Abcr (-/-) mice. Adv Exp Med Biol 664:533-539. 10.1007/978-1-4419-1399-9_61

Wu Y, Zhou J, Fishkin N, Rittmann BE, and Sparrow JR. 2011. Enzymatic degradation of A2E, a retinal pigment epithelial lipofuscin bisretinoid. J Am Chem Soc 133:849-857. 10.1021/ja107195u

Zadlo A, Pilat A, Sarna M, Pawlak A, and Sarna T. 2017. Redox Active Transition Metal ions Make Melanin Susceptible to Chemical Degradation Induced by Organic Peroxide. Cell Biochem Biophys. 10.1007/s12013-017-0793-6

Zareba M, Szewczyk G, Sarna T, Hong L, Simon JD, Henry MM, and Burke JM. 2006. Effects of photodegradation on the physical and antioxidant properties of melanosomes isolated from retinal pigment epithelium. Photochem Photobiol 82:1024-1029.

Zhao Z, Chen Y, Wang J, Sternberg P, Freeman ML, Grossniklaus HE, and Cai J. 2011. Agerelated retinopathy in NRF2-deficient mice. PLoS One 6:e19456. 10.1371/journal.pone.0019456

Zhu X, Wang K, Zhang K, Zhou F, and Zhu L. 2016. Induction of oxidative and nitrosative stresses in human retinal pigment epithelial cells by all-trans-retinal. Exp Cell Res 348:87-94. 10.1016/j.yexcr.2016.09.002

Zigler JS, Jr., Zhang C, Grebe R, Sehrawat G, Hackler L, Jr., Adhya S, Hose S, McLeod DS, Bhutto I, Barbour W, Parthasarathy G, Zack DJ, Sergeev Y, Lutty GA, Handa JT, and Sinha D. 2011. Mutation in the betaA3/A1-crystallin gene impairs phagosome degradation in the retinal pigmented epithelium of the rat. $J$ Cell Sci 124:523-531. $10.1242 / j c s .078790$ 


\section{Figure 1}

Retinal degeneration in Stargardt mice.

(A) Representative light micrographs of 12-month-old pigmented and albino Abca4\% and Abca 4 .Rdh 8 mice. Albino Abca4 $\%$ mice have shorter outer segments whereas $\mathrm{Abca}^{\circ} \%$.Rdh ${ }^{\%}$ mice have hypertrophic RPE. (B) Quantification of outer segment length in Stargardt mice compared to WT animals. In albino Abca 4 mice, outer segment length is already reduced in 4-month-old animals, compared to albino WT. Abca4 $\%$.Rdh 8 - mice show a significant reduction of outer segment length between 3 and 6 months. ( $n=$ on average 2 eyes/age group, * $p \leq 0.001$, Steel-Dwass All Pairs test) (C) Quantification of numbers of photoreceptor nuclei per $100 \mu \mathrm{m}$ width of retina. Linear regression and 95\% confidence interval are shown. ( $n=$ on average 3 eyes/age group). (D) A pyknotic nucleus is present (black arrowhead) next to a cone nucleus (white arrowhead) (albino $A b c a 4^{-}, 4$ months). (E) Photoreceptor nuclei are located next to unusually thinned RPE cells (albino Abca4 ${ }^{-/}, 4$ months). IS: inner segments, OS: outer segments, RPE: retinal pigment epithelium, N: nucleus 

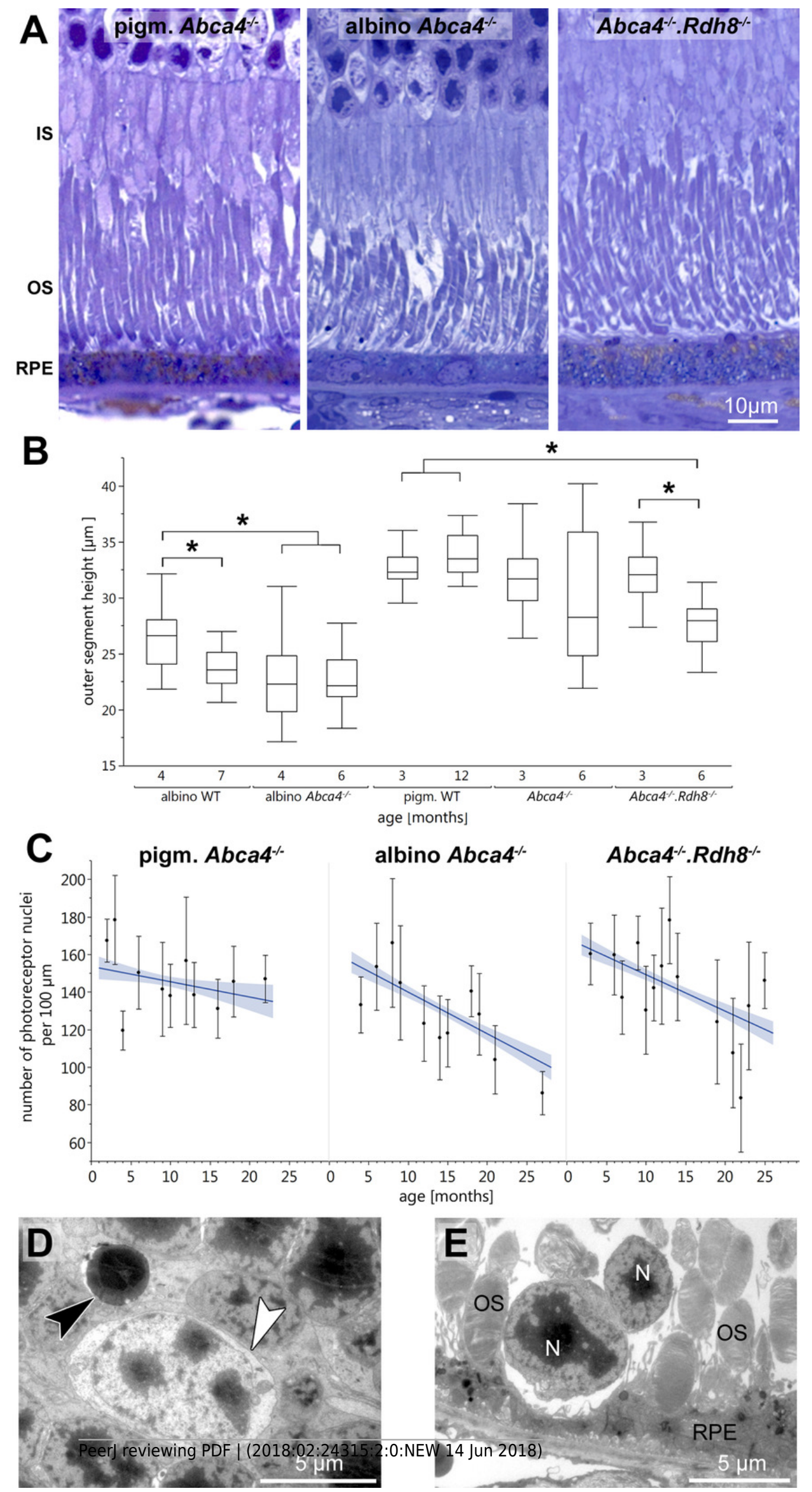


\section{Figure 2}

Morphological changes in inner and outer segments of albino Abca4\% mice.

(A) Disc membrane stacking in outer segments gets more disorganized towards the RPE (albino $\mathrm{Abca}^{-\kappa}, 6$ months). (B) Inner segments are ruptured (arrowheads) (albino $\mathrm{Abca} 4^{-\%}, 4$ months). (C) Electron dense agglomerations (arrowhead) are seen between inner and outer segment (albino Abca4\%, 6 months). (D) Newly synthesized disk membranes are disorganized and accumulate outside of the outer segment (arrowhead) (albino Abca $\%$, 9 months). IS: inner segments, OS: outer segments
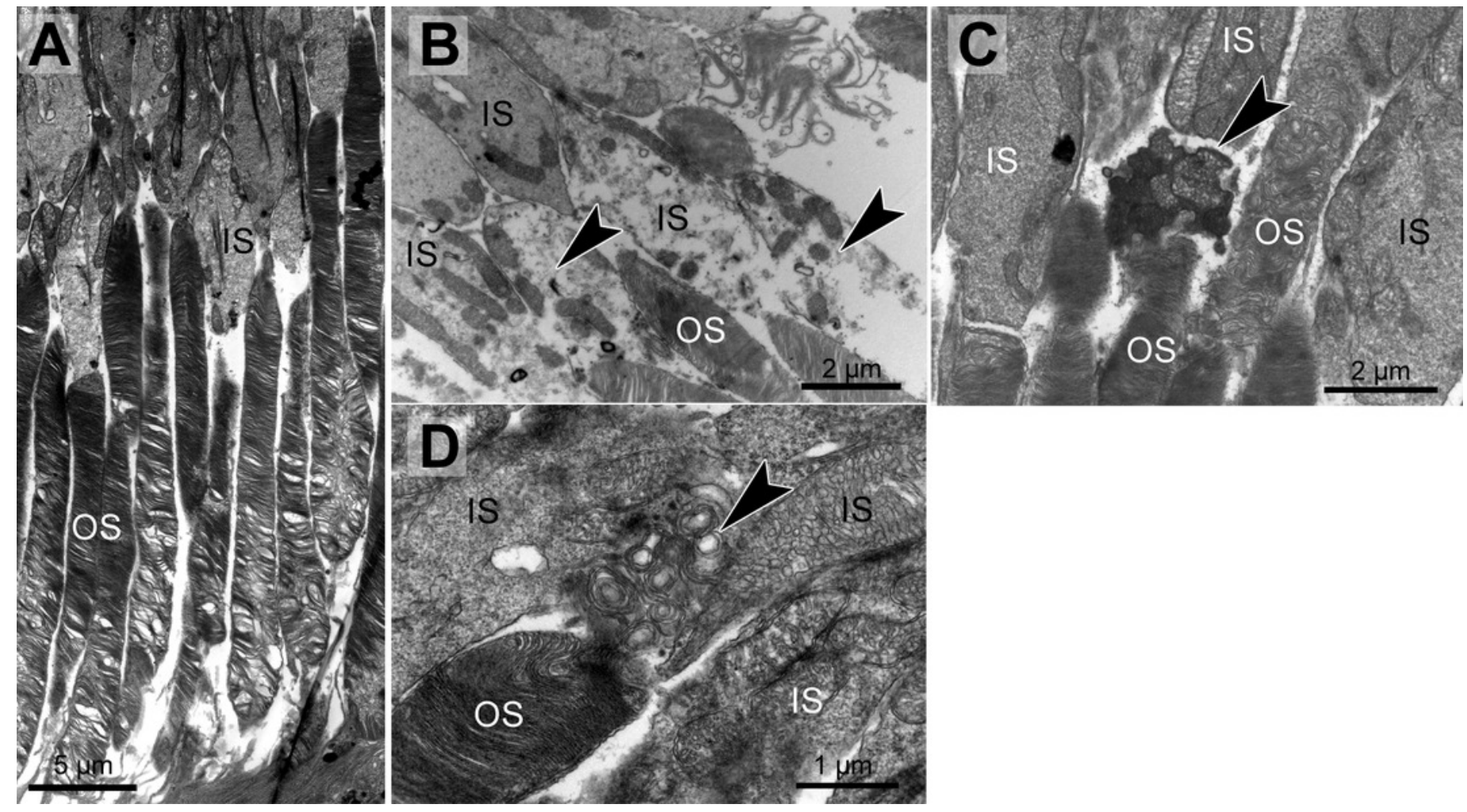


\section{Figure 3}

Vacuole-like structures in RPE cells are most prominent in albino Abca4 $\%$ mice.

(A) In light microscopic images, vacuole-like structures (black arrowheads) within the RPE layer are present in albino Abca4 $4^{-1}$ mice (6 months). (B) A vacuole-like structure (asterisk) turns out to be an enlargement of the intercellular space. Inset: apically, the vacuole-like structure is limited by a junctional complex consisting of a desmosome (arrow), zonula adherens (white arrowhead) and tight junction (black arrowhead) (albino Abca4 ${ }^{-}, 9$ months). (C) Vacuole-like structures are regularly filled with very heterogeneous types of material and are surrounded by a membrane (albino Abca4 $\%, 15$ months). (D) Quantification in electron microscopy revealed that vacuole-like structures are barely present in pigmented $A b c a 4 \%$ and $A b c a 4 \%$ Rdh 8 mice ( $\mathrm{n}=$ on average 2 eyes/age group). OS: outer segments, RPE: retinal pigment epithelium, BM: Bruch's membrane 


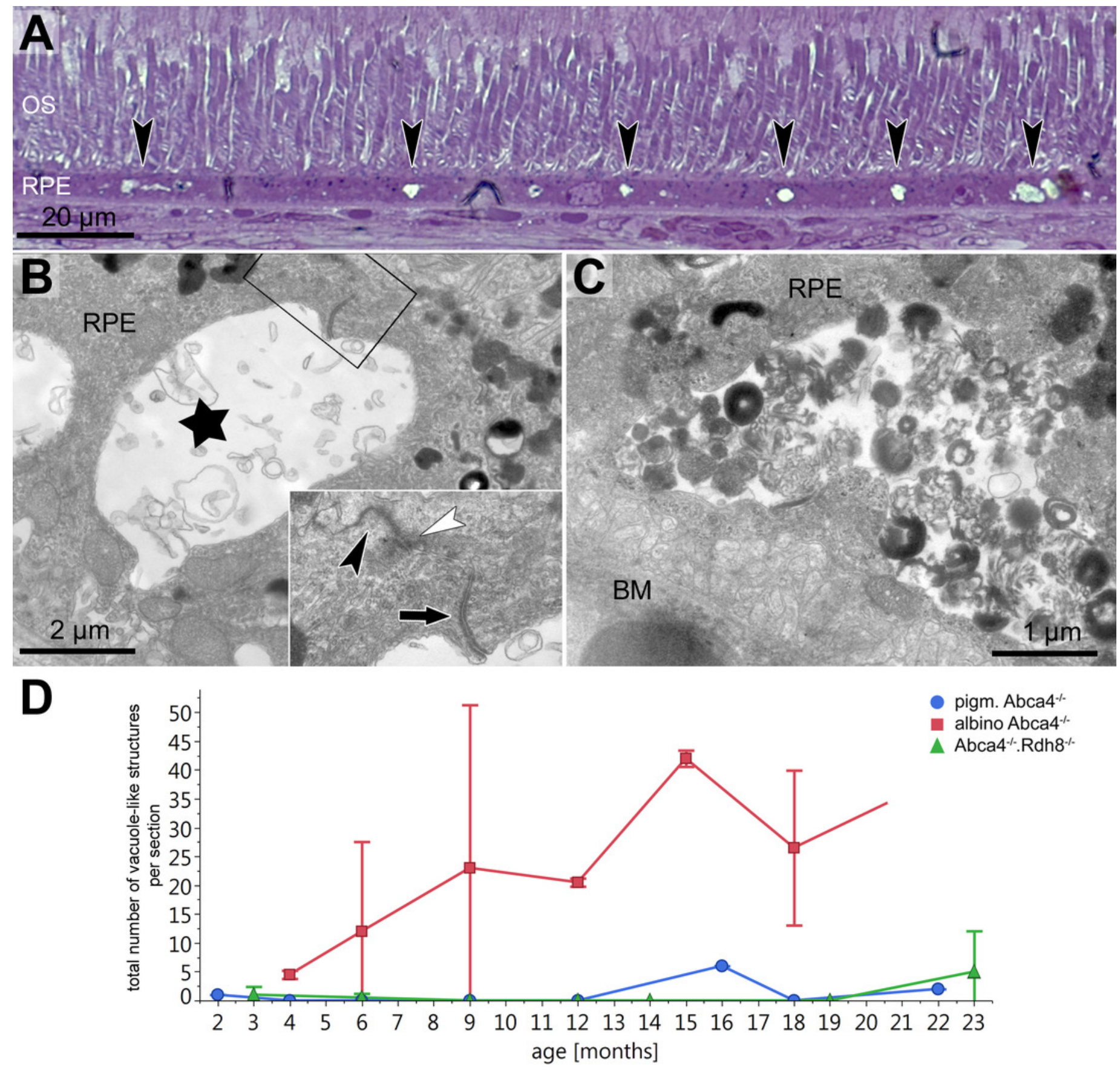




\section{Figure 4}

Lipofuscin in Stargardt mice is highly variable in fluorescence and electron microscopy.

(A) Comparison of lipofuscin autofluorescence in paraffin-embedded sections of 12-month-

old Stargardt mice. (B) Lipofuscin and melanolipofuscin have different morphologies in the different Stargardt mouse strains. In pigmented $A b c a 4 \%$ mice, lipofuscin has a homogenous electron-density and sharp demarcations (black arrowheads), while in albino Abca $4 \%$ mice, most lipofuscin granules have a flocculent electron-density with unclear demarcations (white arrowheads). While in young $A b c a 4 \%$ Rdh $8 \%$ mice, only granules with homogenous electron density can be found, both lipofuscin morphologies can be found in aged animals. M: melanin granules (pigmented and albino Abca4 $\% 9$ months, Abca4 $\%$ Rdh ${ }^{\%}, 12$ months) (C)

Membranes surrounding lipofuscin and melanolipofuscin with flocculent electron density are often damaged (white arrowheads) (Abca $4^{-1}, R d h 8^{-}, 21$ months). (D) In very old Stargardt mice, the RPE cytoplasm is abnormally electron dense. Borders of individual lipofuscin

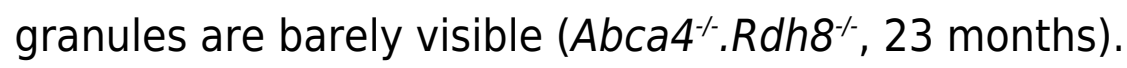



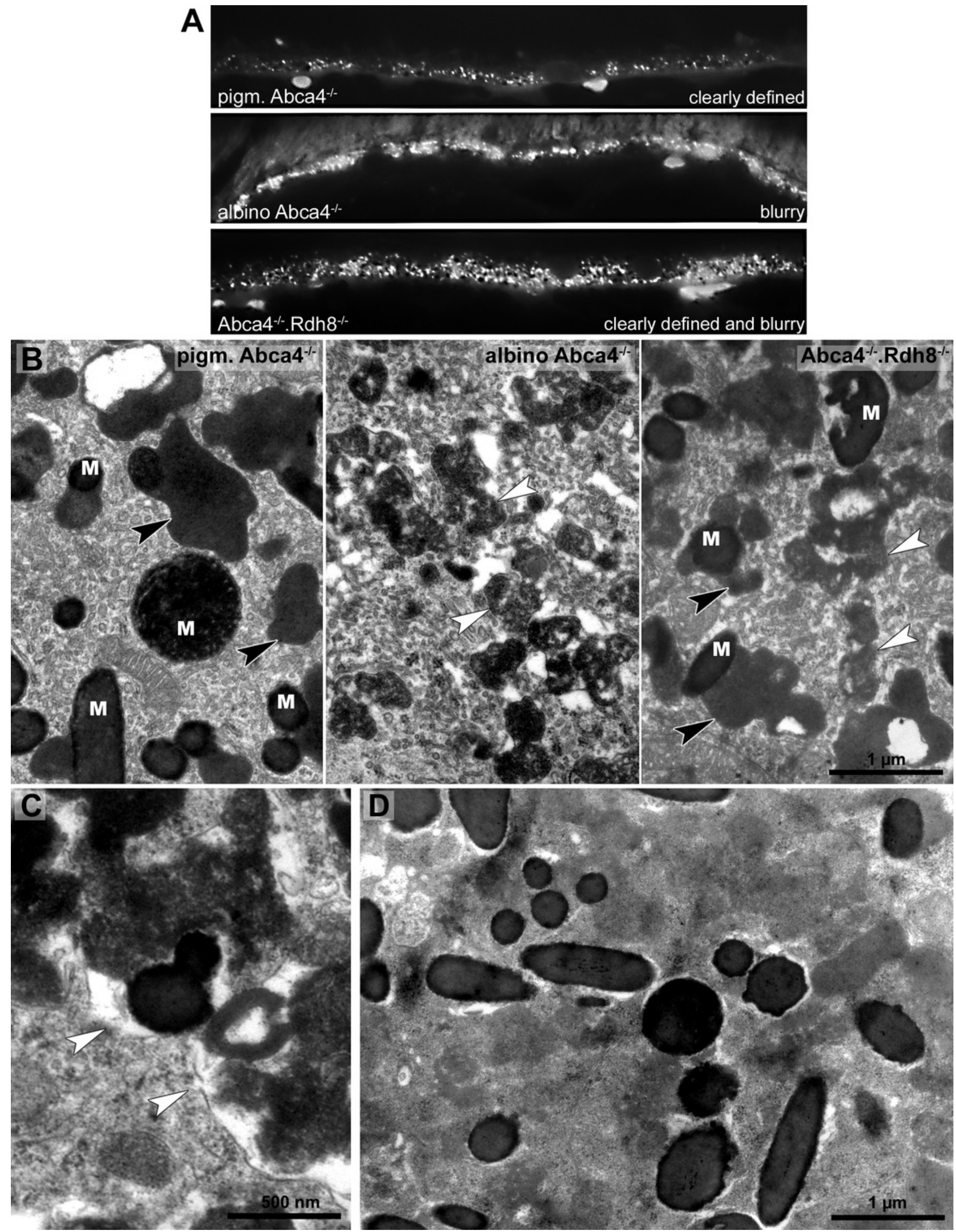
Figure 5

Quantification of the RPE area occupied by lipofuscin in young and old Stargardt mouse strains.

Lipofuscin granules and lipofuscin moieties of melanolipofuscin granules were taken into account. ( $\mathrm{n}=$ on average 5 eyes/age group, $\# \mathrm{p}<0.05, * p<0.0001$, Steel-Dwass All Pairs test).

4-6 months
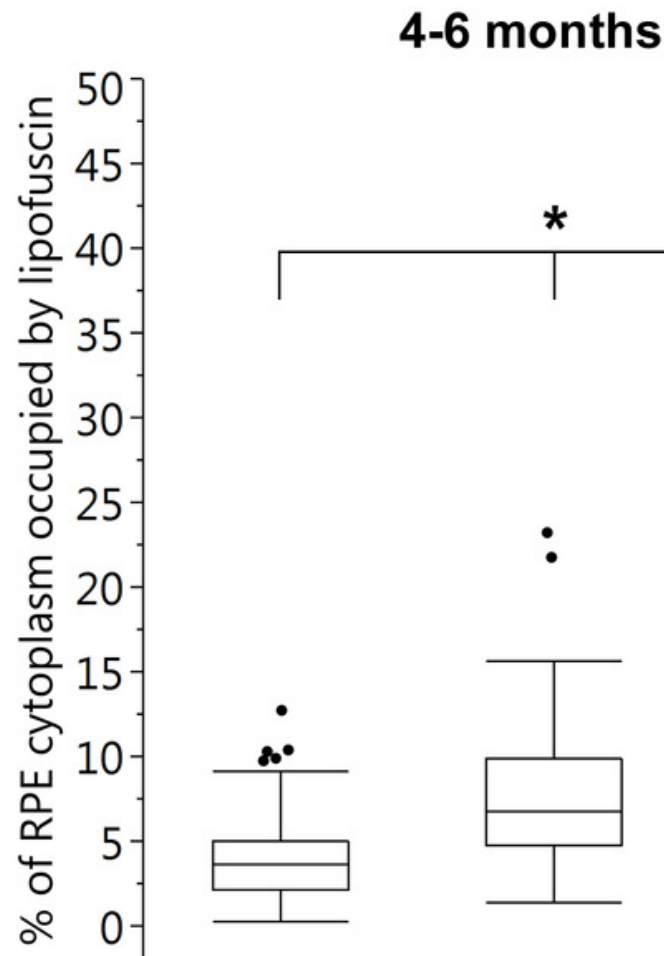

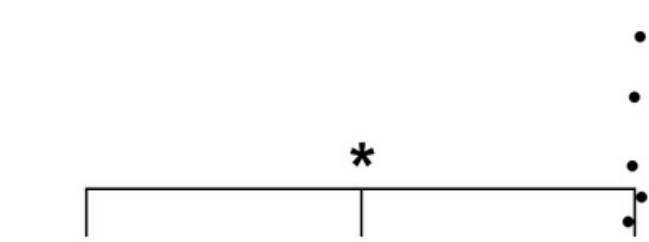

pigm.

Abca4 $^{-/-}$

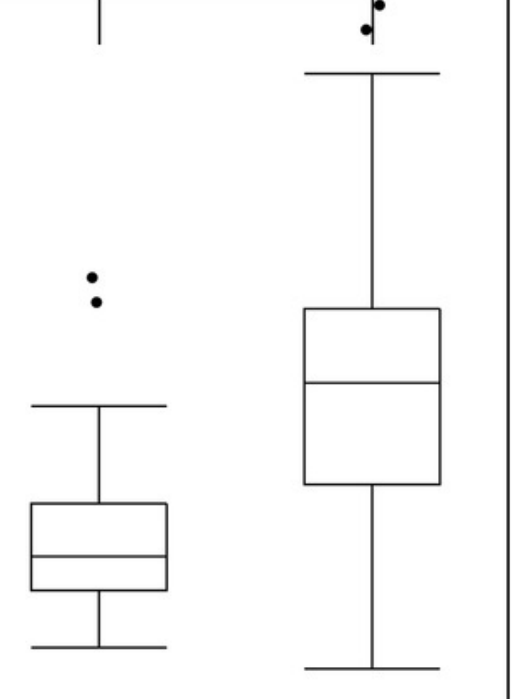

albino Abca4 ${ }^{-/-}$ $\cdot$
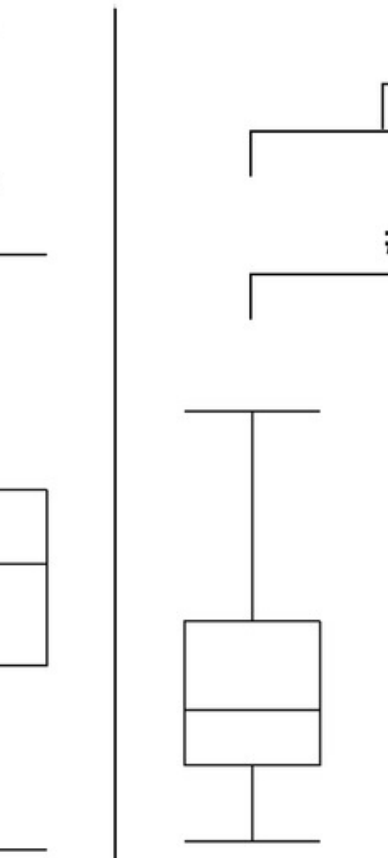

12 months

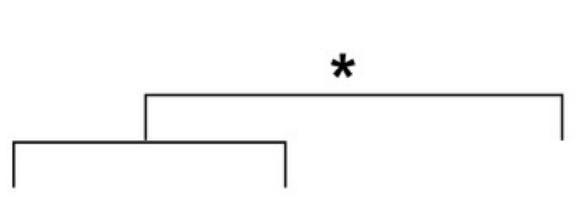

\#
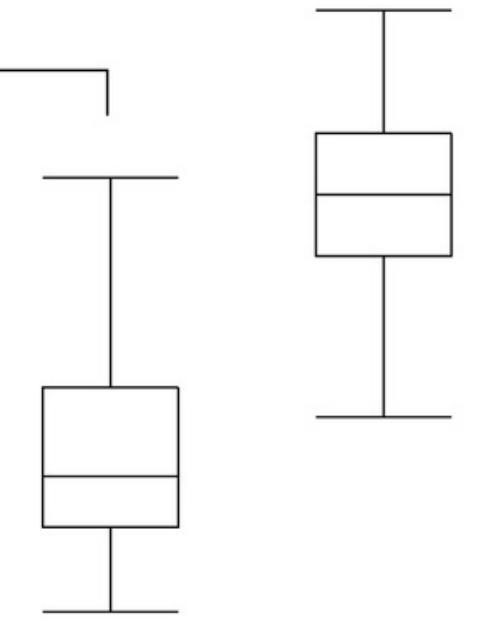

pigm.

Abca4 ${ }^{-/-}$

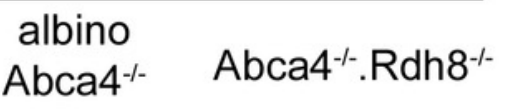




\section{Figure 6}

RPE integrity is compromised in albino Abca 4 mice.

(A) Detached and lysed RPE cell situated in the subretinal space (albino $A b c a 4 \%, 4$ months).

(B) RPE cells of all Stargardt mouse strains regularly contain undigested outer segments in the basal part. Few cells in albino Abca4 $\%$ mice contain several large undigested outer segments (arrowheads) that claimed the majority of the cytoplasm (albino Abca $4^{-1,} 4$ months). (C) Expansion of the basal labyrinth along the lateral cell borders is regularly seen in albino Abca4 $\%$ animals (9 months). Arrowheads indicate the cell border. Basal infoldings were filled with a homogenous material (arrow). (D) Basal labyrinth expansion is reduced in the Stargardt mouse strains compared to the respective wild types. OS: outer segments, RPE: retinal pigment epithelium, BM: Bruch's membrane
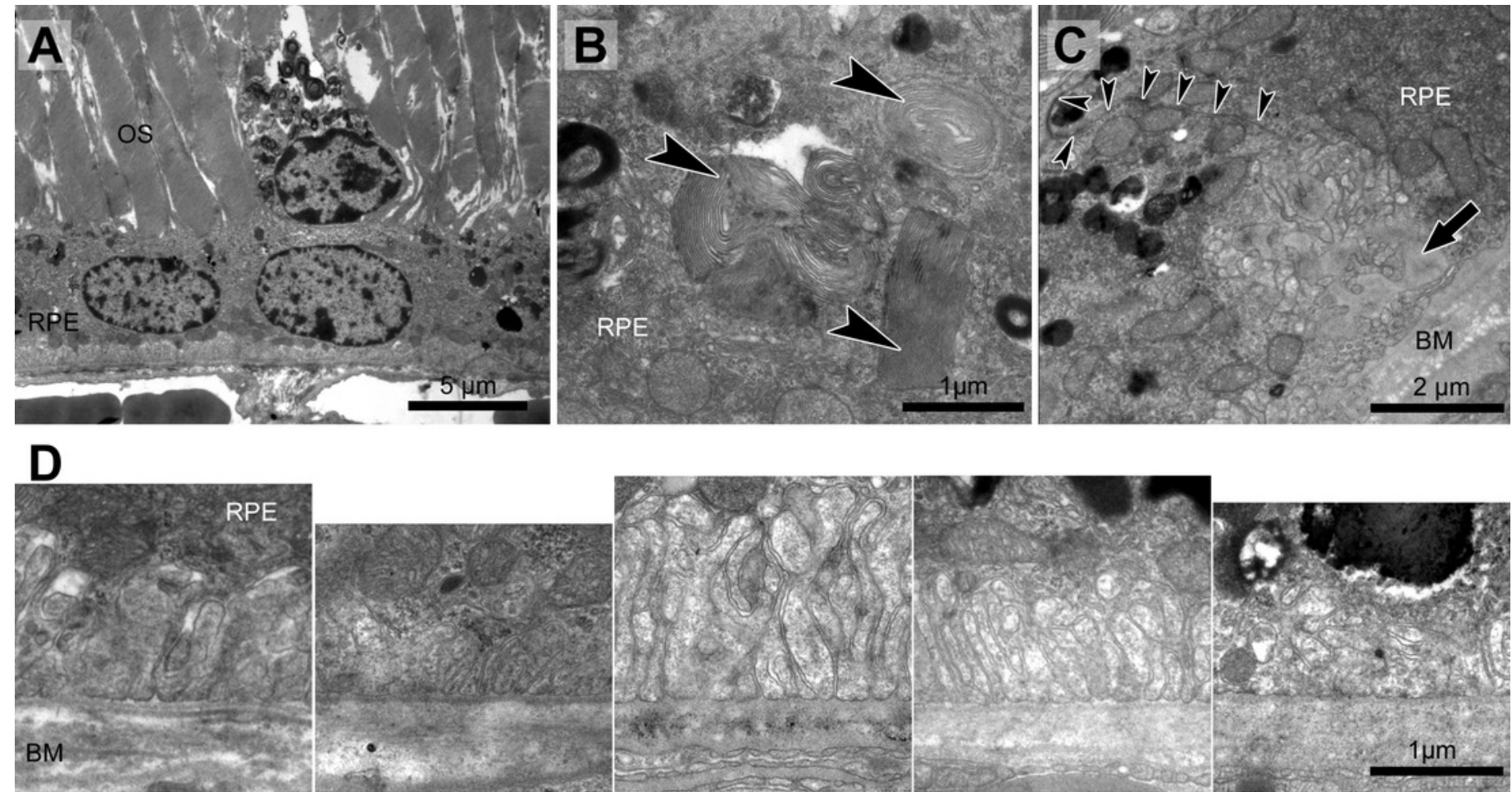

albino WT 7 mo

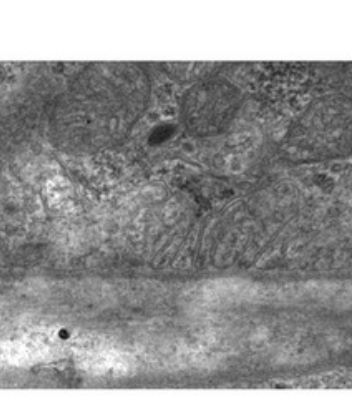

albino $A b c a 4^{\star-~} 6 \mathrm{mo}$

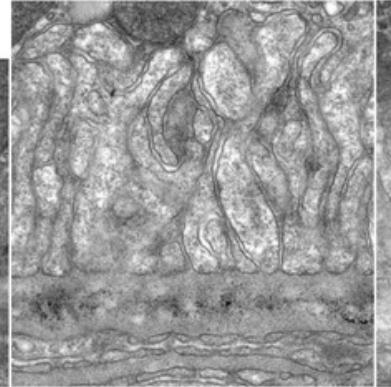

pigm. WT 12 mo

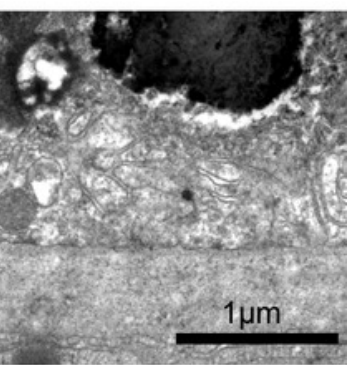

pigm $A b c a 4^{-/} . R_{d h} 8^{-/ 12}$ mo 


\section{Figure 7}

Thickening of Bruch's Membrane is comparable in all Stargardt mouse strains.

(A) Representative electron microscopic images of Bruch's membrane in 12-month-old Stargardt mice. Thickness of Bruch's membrane was measured from the basement membrane of the RPE to the basement membrane of the endothelial cell of the choriocapillaris (indicated by bars). (B) Thickness of Bruch's membrane increases in all Stargardt mice to the same extent. There is no significant difference between the different Stargardt mouse strains. ( $n=$ on average 2 eyes/age group) RPE: retinal pigment epithelium, CC: choriocapillaris
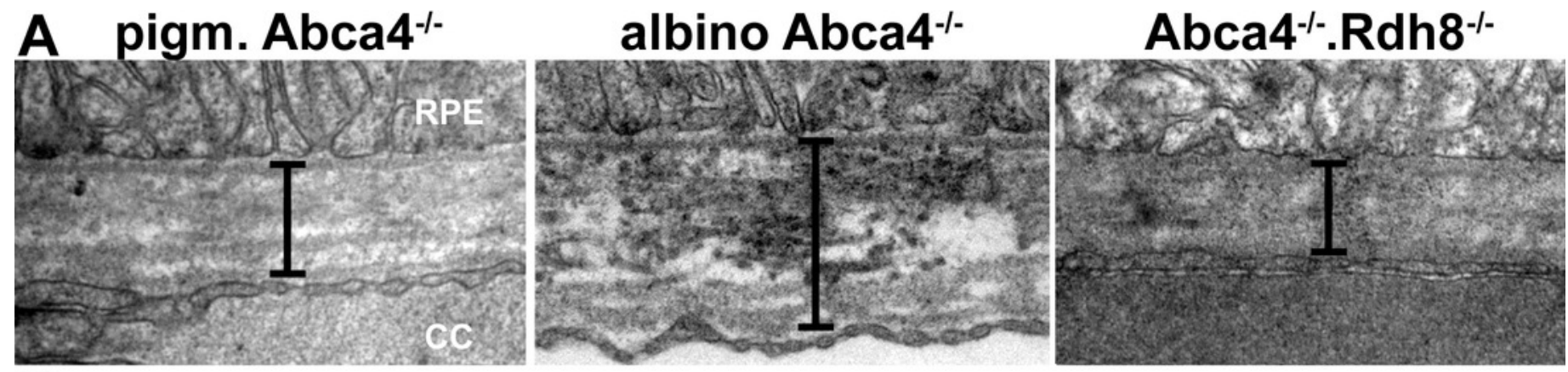

B

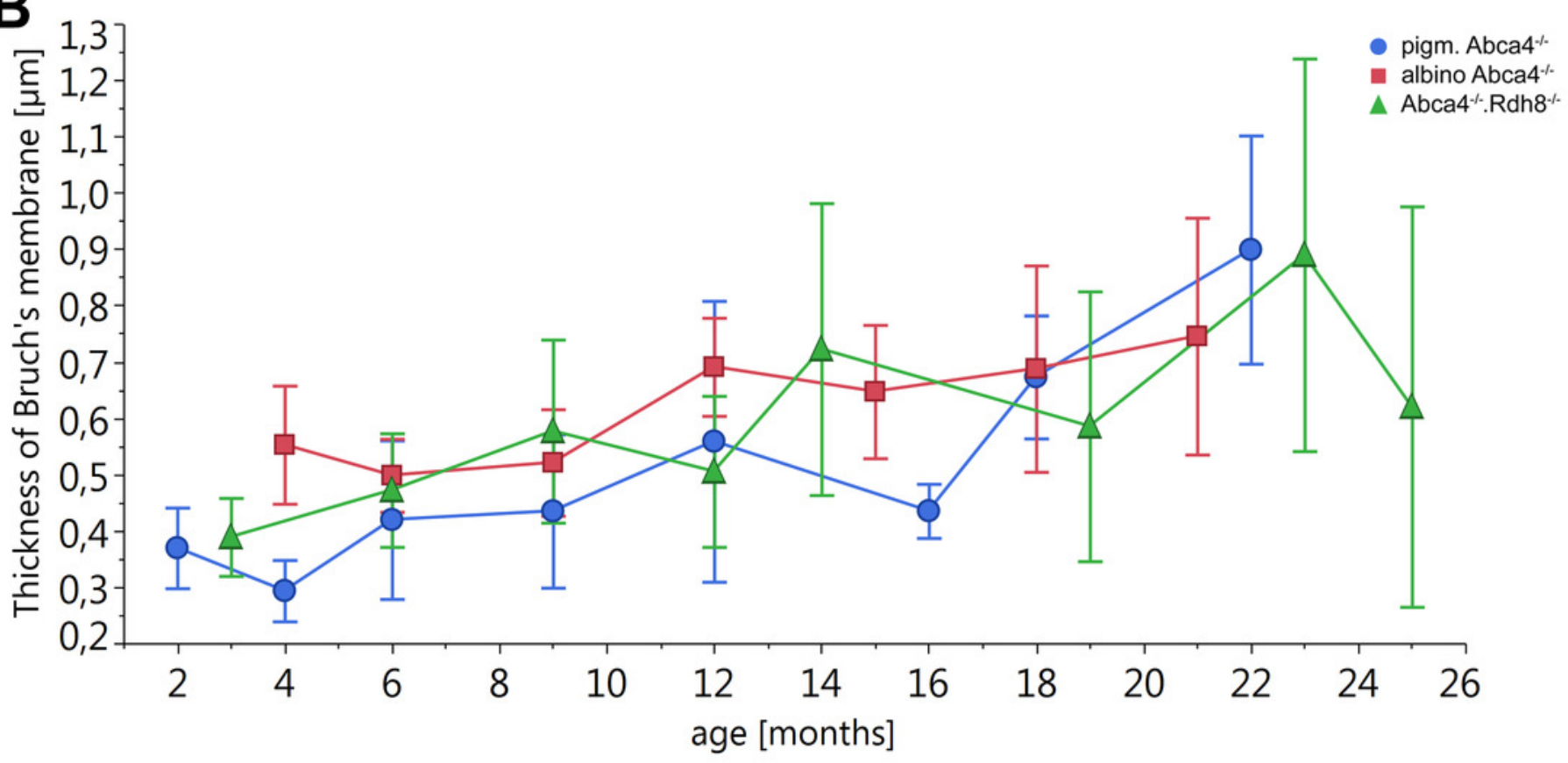




\section{Figure 8}

Histopathology and pigment changes in a human donor eye with late Stargardt disease.

(A) Light microscopic panorama picture of a human donor Stargardt retina: The left area is histologically complete with photoreceptors and a confluent RPE layer. On the right, a typical geographic atrophy with lack of photoreceptors and RPE is present. (B) Electron microscopic overview of the RPE of a human donor eye with Stargardt disease (72 years old). RPE is completely filled with pigment granules. Microvilli and basal infoldings are missing and Bruch's membrane is unorganized and contains electron dense deposits. (C) Big clusters of melanolipofuscin (arrowheads). (D) Fluorescence micrograph of a semi-thin section also shows abundant lipofuscin autofluorescence and cluster formation (arrowhead). INL: inner nuclear layer, ONL: outer nuclear layer, RPE: retinal pigment epithelium, BM: Bruch's membrane, CC: choriocapillaris 


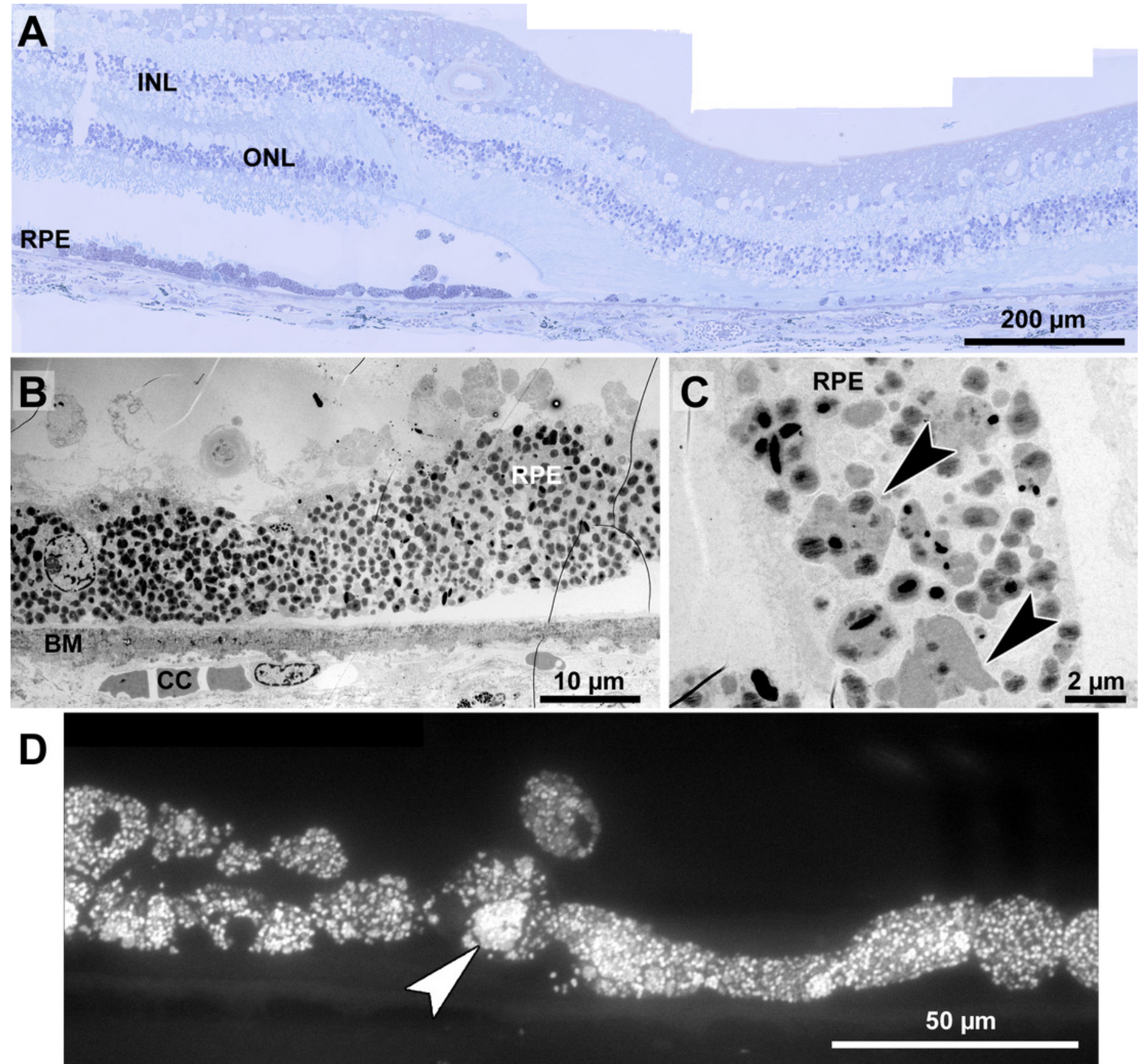




\section{Figure 9}

HNE, MDA and NITT adducts are present in RPE cells of 12-month-old Stargardt mice indicating oxidative stress.

Immunohistochemical stainings show that (A, B) HNE, (C, D) MDA and (E, F) NITT adducts (red) can be found in the RPE of pigmented $A b c a 4^{-\%}$, albino $A b c a 4^{-\%}$, and $A b c a 4^{-\%}, R d h 8^{-\%}$ mice with levels being lowest in pigmented $A b c a 4^{-/}$mice. A, $\mathbf{C}$ and $\mathbf{E}$ show negative controls (primary antibody was omitted) for each antibody; B, D and F show the respective antibody stain for each primary antibody.

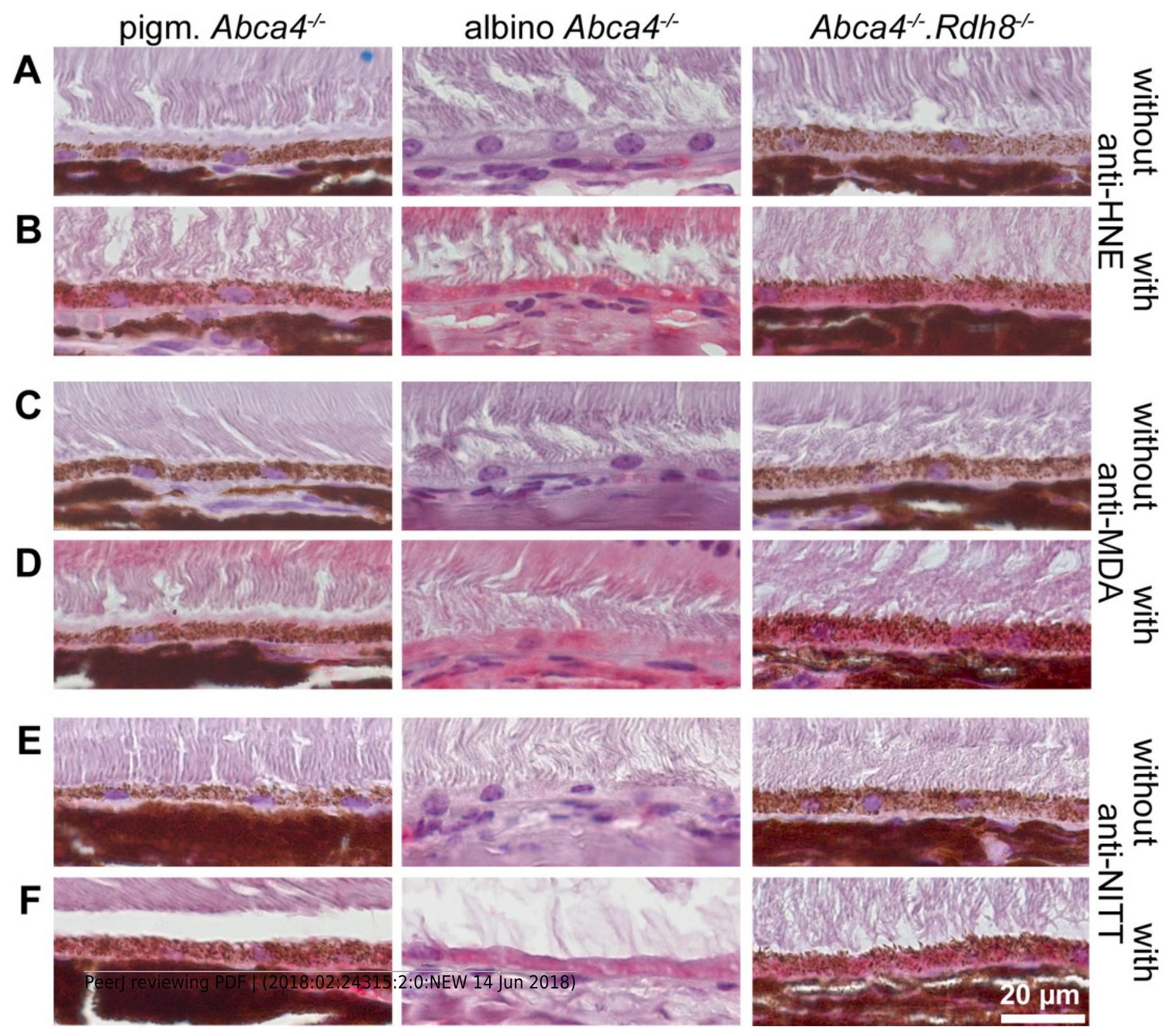




\section{Figure 10}

Both melanin from S. officinalis and porcine RPE melanin can quench superoxide radicals in an NBT assay.

(A) Sepia melanin shows a dose dependent capability to quench superoxide anions produced by light-illuminated riboflavine. ( $n=8$ per group, two independent experiments, * $p$ $<0.05$, Steel-Dwass All Pairs test). (B) Porcine RPE melanin (concentration $3 \times 10^{4}$ granules $/ \mathrm{ml}$ ) quenches superoxide anions. Negative control contained all reagents but no melanin and was kept in the dark so no superoxide was produced ( $n=12$ per group, three independent experiments, $* p<0.05$, Steel-Dwass All Pairs test).
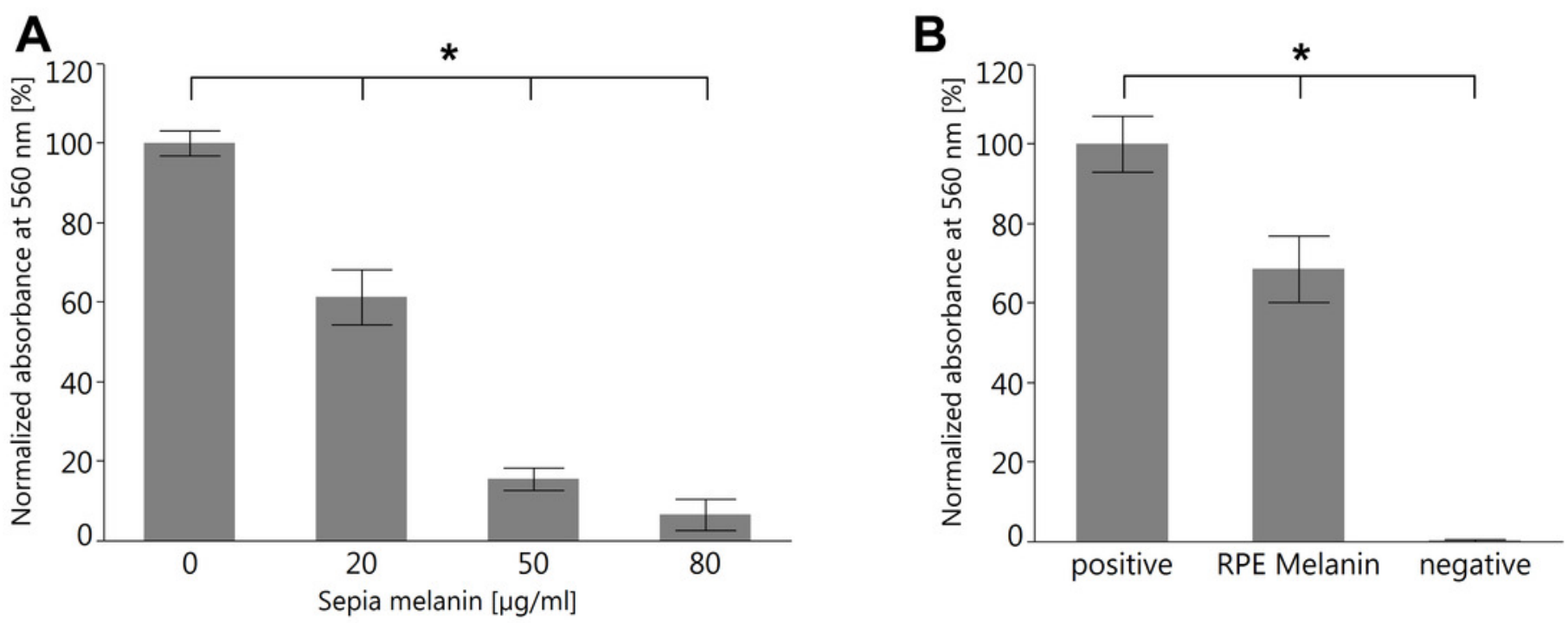


\section{Table 1 (on next page)}

Ultrastructural observations in WT and Stargardt mice. 


\begin{tabular}{|c|c|c|c|c|c|c|c|c|c|c|c|c|c|c|c|c|c|c|c|c|c|c|c|c|c|c|c|}
\hline \multirow[b]{2}{*}{ Age [months] } & \multicolumn{2}{|c|}{$\begin{array}{c}\text { albino } \\
\text { WT }\end{array}$} & \multicolumn{7}{|c|}{ albino $A b c a 4^{-/-}$} & \multicolumn{2}{|c|}{$\begin{array}{l}\text { pigm. } \\
\text { WT }\end{array}$} & \multicolumn{8}{|c|}{ pigm. $A b c a 4^{-/-}$} & \multicolumn{8}{|c|}{$A b c a 4^{-/-} \cdot R d h 8^{-/-}$} \\
\hline & 4 & 7 & 4 & 6 & 9 & 12 & 15 & 18 & 21 & 3 & 12 & 2 & 4 & 6 & 9 & 12 & 16 & 18 & 22 & 3 & 6 & 9 & 12 & 14 & 19 & 23 & 25 \\
\hline $\begin{array}{l}\text { OS disks } \\
\text { disarranged towards } \\
\text { the tip }\end{array}$ & - & $+/-$ & $+/-$ & + & + & + & + & + & + & - & - & - & - & - & - & - & - & - & - & - & $+/-$ & $+/-$ & + & $+/-$ & $+/-$ & $+/-$ & $+/-$ \\
\hline $\begin{array}{l}\text { OS disks } \\
\text { disarranged along } \\
\text { the whole axis } \\
\end{array}$ & - & - & - & $+/-$ & + & + & + & + & + & - & - & - & - & - & - & - & - & - & - & - & $+/-$ & $+/-$ & + & + & + & + & + \\
\hline Swollen/ruptured IS & - & - & + & + & + & + & + & + & + & - & - & - & - & - & $+/-$ & - & - & - & - & - & - & - & $+/-$ & - & - & - & - \\
\hline $\begin{array}{l}\text { Presence of } \\
\text { lipofuscin: }\end{array}$ & & & & & & & & & & & & & & & & & & & & & & & & & & & \\
\hline $\begin{array}{l}\text { - homogenous } \\
\text { electron density }\end{array}$ & $+/-$ & $+/-$ & + & + & $+/-$ & + & + & + & + & $+/-$ & + & + & ++ & ++ & +++ & ++++ & ++++ & +++ & +++ & + & ++ & + & ++++ & +++ & +++ & + & ++ \\
\hline $\begin{array}{l}\text { - flocculent } \\
\text { electron density }\end{array}$ & $+/-$ & $+/-$ & + & ++ & +++ & +++ & +++ & +++ & +++ & $+/-$ & $+/-$ & - & - & - & - & - & - & - & - & - & - & - & $+/-$ & +++ & ++ & ++ & ++ \\
\hline $\begin{array}{l}\text { Number of lipid } \\
\text { droplets: }\end{array}$ & & & & & & & & & & & & & & & & & & & & & & & & & & & \\
\hline - apical & ++ & ++ & $+/-$ & - & - & - & $+/-$ & - & - & + & ++ & $+/-$ & - & - & - & - & - & - & - & - & - & - & - & - & - & - & - \\
\hline - basal & - & ++ & + & ++ & ++ & + & + & + & $+/-$ & + & ++ & + & $+/-$ & $+/-$ & + & $+/-$ & - & $+/-$ & - & $+/-$ & + & $+/-$ & - & $+/-$ & $+/-$ & $+/-$ & $+/-$ \\
\hline $\begin{array}{l}\text { Detached RPE cells } \\
\text { in subretinal space }\end{array}$ & $+/-$ & + & + & + & $+/-$ & + & + & $+/-$ & $+/-$ & - & - & - & - & - & - & - & - & $+/-$ & - & - & - & - & - & $+/-$ & + & + & + \\
\hline $\begin{array}{l}\text { Undegraded } \\
\text { phagosomes in basal } \\
\text { part of RPE }\end{array}$ & - & + & + & $+/-$ & + & + & + & ++ & + & $+/-$ & + & + & + & + & $+/-$ & + & + & + & + & + & ++ & +++ & + & + & + & + & ++ \\
\hline $\begin{array}{l}\text { Expansions of the } \\
\text { basal labyrinth along } \\
\text { the cell border }\end{array}$ & $+/-$ & + & + & + & + & + & + & + & + & - & - & - & - & - & - & - & - & - & - & - & - & - & - & - & - & + & - \\
\hline
\end{tabular}

- $\quad$ not or rarely observed

+ occasionally observed

$+\quad$ regularly observed

$++\quad$ regularly observed in high amounts

$++\quad$ regularly observed in extreme amounts 